\title{
\begin{tabular}{l|l} 
Mibraries & DSpace@MIT
\end{tabular}
}

\author{
MIT Open Access Articles
}

A Microfabricated Planar Electrospray Array
Ionic Liquid lon Source With Integrated Extractor

The MIT Faculty has made this article openly available. Please share how this access benefits you. Your story matters.

Citation: Gassend, B. et al. "A Microfabricated Planar Electrospray Array lonic Liquid Ion Source With Integrated Extractor." Microelectromechanical Systems, Journal of 18.3 (2009): 679-694. () 2009 IEEE

As Published: http://dx.doi.org/10.1109/JMEMS.2009.2015475

Publisher: Institute of Electrical and Electronics Engineers

Persistent URL: http://hdl.handle.net/1721.1/59347

Version: Final published version: final published article, as it appeared in a journal, conference proceedings, or other formally published context

Terms of Use: Article is made available in accordance with the publisher's policy and may be subject to US copyright law. Please refer to the publisher's site for terms of use. 


\section{A Microfabricated Planar Electrospray Array Ionic Liquid Ion Source With Integrated Extractor}

Blaise Gassend, Luis Fernando Velásquez-García, Akintunde Ibitayo Akinwande, and Manuel Martínez-Sánchez

\begin{abstract}
This paper reports the design, fabrication, and experimental characterization of a fully microfabricated planar array of externally fed electrospray emitters that produces heavy molecular ions from the ionic liquids $\mathrm{EMI}_{-\mathrm{BF}_{4}}$ and EMI-Im. The microelectromechanical systems (MEMS) electrospray array is composed of the following two microfabricated parts: 1) an emitter die with as many as 502 emitters in $1.13 \mathrm{~cm}^{2}$ and 2) an extractor component that provides assembly alignment, electrical insulation, and a common bias voltage to the emitter array. The devices were created using Pyrex and silicon substrates, as well as microfabrication techniques such as deep reactive ion etching, low-temperature fusion bonding, and anodic bonding. The emitters are coated with black silicon, which acts as a wicking material for transporting the liquid to the emitter tips. The extractor electrode uses a 3-D MEMS packaging technology that allows hand assembly of the two components with micrometer-level precision. Experimental characterization of the MEMS electrospray array includes current-voltage characteristics, time-of-flight mass spectrometry, beam divergence, and imprints on a collector. The data show that with both ionic liquids and in both polarities, the electrospray array works in the pure ionic regime, emitting ions with as little as $500 \mathrm{~V}$ of bias voltage. The data suggest that the MEMS electrospray array ion source could be used in applications such as coating, printing, etching, and nanosatellite propulsion.

[2008-0270]
\end{abstract}

Index Terms-Black silicon, electrospray array, ion source, ionic liquid, 3-D packaging.

\section{INTRODUCTION}

$\mathbf{E}$ LECTROSPRAY refers to the technique of ionizing electrically conductive liquids using high electrostatic fields. If an electrically conductive liquid is under the influence of an electric field, the field will produce electrostatic pressure on the free surface of the liquid (meniscus). The surface tension of the liquid tends to counteract the effect of the electrostatic pressure. For sufficiently small bias voltages, the perturbation of the

Manuscript received October 31, 2008; revised January 16, 2009. First published March 16, 2009; current version published June 3, 2009. This work was supported in part by the Air Force Office of Scientific Research (M. Birkan, Manager), in part by the Space and Naval Warfare Systems Center through Award N66001-04-1-8925 (Manager R. Nguyen), and in part by the Defense Advanced Research Projects Agency (DARPA)/Microsystems Technology Office and the U.S. Army Soldier Systems Center, Natick, MA, under Contract W911QY-05-1-0002 (DARPA Program Managers C. Nguyen and D. Polla and Army Program Manager H. Girolamo). The work of B. Gassend was supported by Prof. S. Devadas. Subject Editor D. L. DeVoe.

B. Gassend was with the Department of Electrical Engineering and Computer Science, Massachusetts Institute of Technology, Cambridge, MA 02139 USA. He is now with Exponent, Inc., Menlo Park, CA 94025 USA.

L. F. Velásquez-García, A. I. Akinwande, and M. Martínez-Sánchez are with the Massachusetts Institute of Technology, Cambridge, MA 02139 USA (e-mail: 1fvelasq@mit.edu).

Color versions of one or more of the figures in this paper are available online at http://ieeexplore.ieee.org.

Digital Object Identifier 10.1109/JMEMS.2009.2015475 equilibrium shape of the meniscus remains small. If the electric field is larger than a certain threshold, the meniscus snaps into a conic shape called a Taylor cone [1]. The conic surface equilibrates the surface tension effects and the electrostatic pulling [2], i.e.,

$$
\frac{1}{2} \varepsilon_{o} E_{n}^{2}=\frac{\gamma \cdot \cot \left[\alpha_{T}\right]}{r}
$$

where $E_{n}$ is the normal electric field acting on a point on the cone surface, $\varepsilon_{o}$ is the permittivity of free space, $\gamma$ is the surface tension of the liquid, $r$ is the distance between the point and the cone tip or apex, and $\alpha_{T}$ is the Taylor cone semiangle. Near the apex, the electric field is strong enough to cause charged particle emission. The mechanism through which particles are emitted at the tip of the Taylor cone depends on the regime of operation, which is influenced by the electrical properties of the liquid and the flow rate. For moderately conductive liquids and large flow rates, the cone emits a jet, which breaks up into charged droplets [2], [3]. For liquids with high enough electrical conductivity and small enough flow rates, the electrospray plume is composed of ions [4]. For intermediate flow rates and electrical conductivities, a mix of ions and droplets is also possible [5]. Field-enhancing structures such as capillary tubes or sharp tips are used as emitters to generate the strong electric fields needed for the electrospray process and to anchor the Taylor cones [6], [7].

Numerous applications of electrospray have been reported. The most successful application for electrospray so far is as ion source for mass spectrometry of large biomolecules, which resulted in a Nobel Prize in Chemistry for J. Fenn in 2002 [8]. Other applications of electrospray in the jet-emitting mode include fuel atomization in combustors [9], [10], nanoparticle generation [11], aerosol generation [12], fiber formation by electrospinning [13] (in this case, the jet does not break up into droplets), crop spraying [14], spray painting [15], polymer coating [16], etching [17], printing [18], and satellite propulsion [3]. There have also been several reports of electrospray devices that work in the ion regime, including liquid metal [19], [20] and ionic liquid ion sources [21] and more specific applications such as mass-efficient nanosatellite engines [22], [23]. Electrospray of ionic liquids enjoys a special interest because of the remarkable properties of these substances. Ionic liquids are molten ionic salts (many of them at room temperature), composed of discrete heavy cations and anions that can be individually customized, with tunable physical, chemical, and biological properties [24].

The ionization of liquids using electrospray has several advantages compared to other approaches such as chemical 

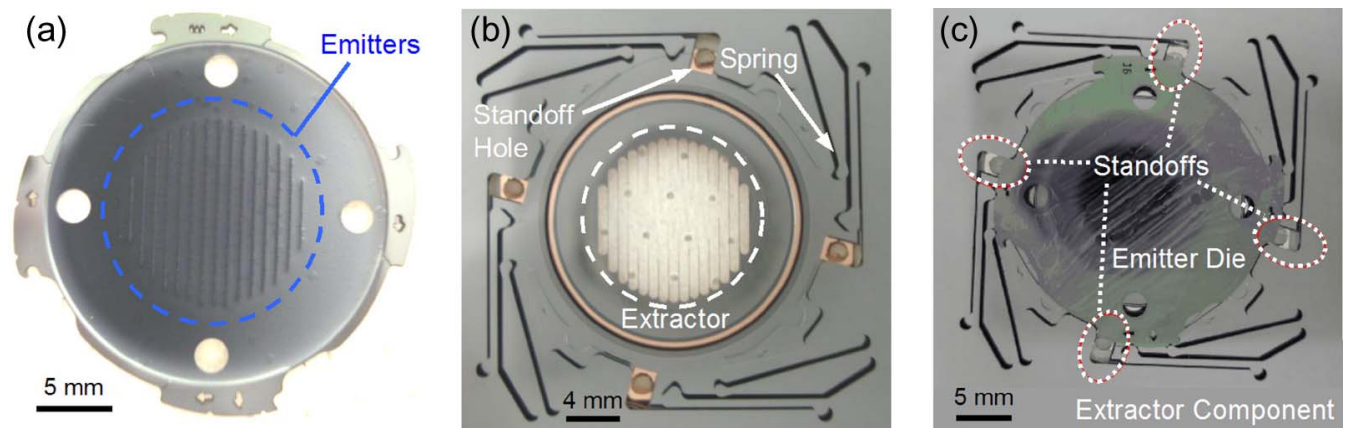

Fig. 1. (a) Microfabricated emitter array die. (b) Extractor component. (c) Backside view of an assembled electrospray array. A set of eight deflection springs surrounding the extractor interacts with the border of the emitter die to assemble the two parts with micrometer-level accuracy, and a set of four standoffs sets the vertical emitter die-to-extractor separation.

ionization and electron impact ionization. For example, when working in the single Taylor cone regime, the electrospray beam has small divergence and low polydispersity [2]. Furthermore, electrospray is capable of bipolar emission [25]. Moreover, electrospray can work in a very stable fashion, delivering repeatable and controllable currents [3]. In addition, the electrospray phenomenon scales favorably with a smaller emitter size. If the dimensions of the emitter are scaled down, the startup voltage [26], [27] and the vaporization losses decrease [26]. The scaling down of electrospray emitters enables batch fabrication, which makes higher emitter packing density possible while lowering the cost per emitter. In particular, plasma-based microfabrication techniques can produce emitter arrays with high emitter uniformity [23].

Electrospray ionization poses several technical challenges for a number of applications. First, the steady-state current and flow rate of one emitter are rather small (usually less than $0.1 \mu \mathrm{A}$ and $10^{-13} \mathrm{~m}^{3} / \mathrm{s}$, respectively), thus requiring emitter multiplexing to reach larger net currents and flow rates. Second, the implementation of multiplexed electrospray devices require flow ballasting structures to control the emission coming from each emitter and achieve uniform array operation [12], [28], [29]. Third, the emitter density is controlled by the extractor aperture, the emitter-to-extractor alignment, and the emitterto-extractor gap. Electrospray requires high electric fields to operate, which usually implies high voltages. High voltages pose electrical insulation difficulties in microelectromechanical systems (MEMS)-based electrospray, including potential shorting due to liquid flooding, and reduction of the vacuum level near the emitter due to the liquid vapor pressure (vacuum applications) [26], [30]-[32].

A substantial amount of work on MEMS-based electrospray has been reported, covering both single- and multiple-emitter sources for diverse applications such as droplet- and ion-based spacecraft engines [23], [28], [31]-[36], droplet-based combustors [10], [12], and droplet-based mass spectrometry [29], [37]-[42]. This paper reports a MEMS planar array of externally fed emitters with an integrated extractor electrode that is based on previously reported work by our group [23]. The device uses the ionic liquids EMI-BF 4 and EMI-Im to produce ions in vacuum. The choice of these liquids is motivated by their very low vapor pressure and their large electrical conductivity at room temperature [43], [44]. To the best of the authors' knowledge, this paper represents the first report of a microfabricated planar electrospray array with an integrated extractor electrode that demonstrates stable operation in the pure ion emission regime. Previously reported devices were linear arrays [28], did not have an integrated MEMS extractor electrode [12], [23], or were not shown to emit only ions. In addition, this MEMS electrospray array uses a flexible modular architecture in which the emitter die is reversibly assembled to the extractor using a system of deflection springs [45], [46]. The assembly system allows testing of a range of quick-to-fabricate emitter dies with the same extractor component, which is the part of the device that incorporates most of the fabrication complexity. This approach contrasts with monolithic emitter-extractor electrospray implementations that require the fabrication of a whole new device to iterate one of its modules and that can only be inspected through destructive means after testing [33], [47]. Section II describes the design of the electrospray array ion source, while Section III elaborates on its fabrication. Finally, Section IV reports and discusses the experimental characterization of the MEMS electrospray array, as well as points out potential applications of this technology.

\section{Description of the Electrospray Array Design}

The MEMS electrospray array is composed of the following two parts: 1) an emitter die [Fig. 1(a)] and 2) an extractor component [Fig. 1(b)]. A set of eight slender deflection springs on the extractor component is used to align and clamp the emitter die into place. The emitter die is about $2.1 \mathrm{~cm}$ in diameter, and the springs are about $1.3 \mathrm{~cm}$ long. The separation distance between the emitter die and the common extractor component is controlled by a set of four standoffs [see Fig. 1(c) and Section II-C]. The design of the electrospray array results from taking into consideration issues such as the following: 1) the hydraulic impedance required for ion emission; 2) the emitter and extractor geometries needed to decrease the startup voltage while increasing the emitter density; 3) the structural resistance of the extractor to electrostatic loads; and 4) the electrode-toextractor assembly scheme to achieve low beam interception and electrical insulation, including electrical breakdown resistance against surface contamination, beam interception, and liquid flooding. 


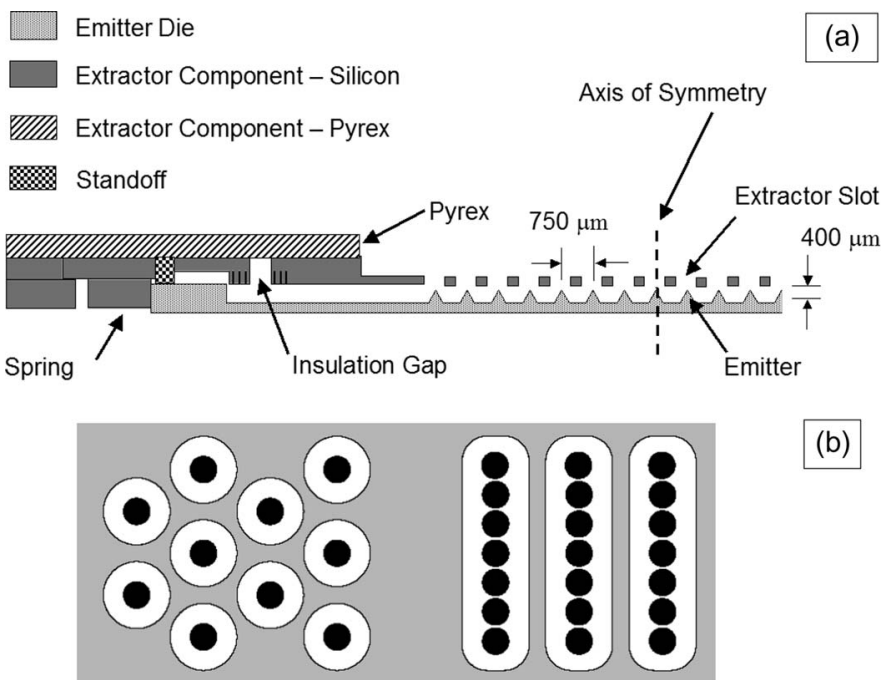

Fig. 2. (a) Cross section of the microfabricated electrospray array ion source. (b) Emitters organized in rows to increase the emitter density.

TABLE I

DESCRIPTION OF THE EMITTER Dies That WeRE BUILT

\begin{tabular}{|l|c|c|c|}
\hline Number of emitters & 1 & 216 & 502 \\
\hline Tip-to-tip spacing $(\mu \mathrm{m})$ & - & 500 & 275 \\
\hline Row spacing $(\mu \mathrm{m})$ & - & 750 & 750 \\
\hline Number of rows & - & 13 & 15 \\
\hline Emitter density $\left(\mathrm{emitters} / \mathrm{cm}^{2}\right)$ & - & 227 & 445 \\
\hline
\end{tabular}

\section{A. Emitter Die}

The emitter die has an array of emitters about $400 \mu \mathrm{m}$ tall, arranged in 13 parallel rows spaced $750 \mu \mathrm{m}$ apart [Fig. 2(a)]. The active area of the emitter die is surrounded by a raised support ring [visible in Fig. 1(a)] that strengthens the emitter die and interfaces with the spring tips in the assembly. The emitters are organized in rows that face a common extractor slot to increase the emitter density, compared to an emitter array where each emitter has an individual concentric extractor [Fig. 2(b)]. Three versions of the emitter die were built, with parameters described in Table I. Emitter dies with as many as 502 emitters in $1.13 \mathrm{~cm}^{2}$ were fabricated. To the best of the authors' knowledge, this paper reports the largest and densest working electrospray array with integrated extractor to date (4.45 emitters $/ \mathrm{mm}^{2}$, almost twice the emitter density reported by Deng et al. [10]). However, Krpoun et al. reported the fabrication of electrospray arrays with integrated extractor that have larger emitter densities (about 18 emitters $/ \mathrm{mm}^{2}$, although only data from one- and two-emitter arrays were reported) [33], and our group previously reported larger and denser working electrospray arrays with no integrated electrode (1024 emitters in $1 \mathrm{~cm}^{2}$ ) [23].

The electrospray emitters are externally fed [Fig. 3(a)]. In this feeding scheme, the liquid is transported to the emitter tip across the surface of the emitter. At the emitter tip, the liquid is then exposed to a high electrostatic field created by
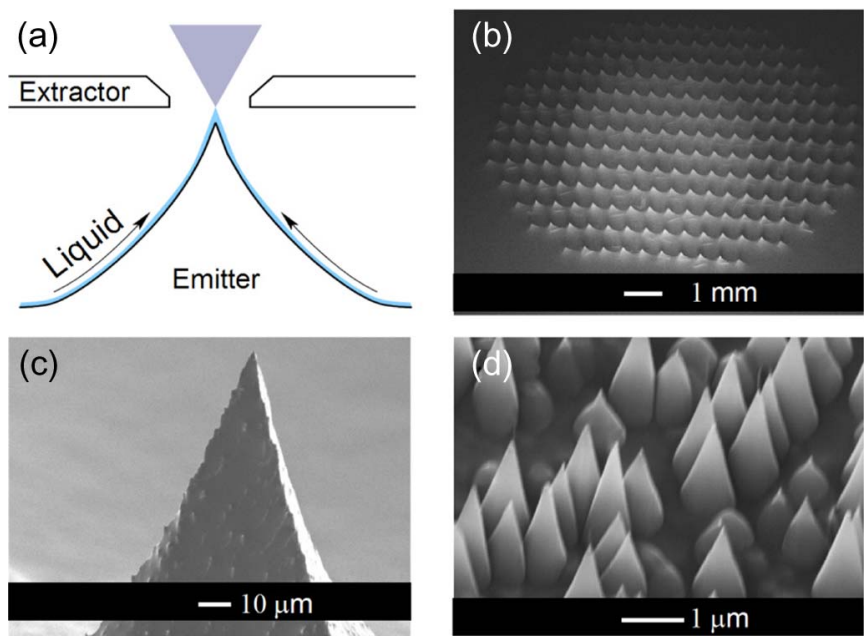

Fig. 3. (a) Schematic of an externally fed emitter. (b) Field view of a monolithic 216-emitter array. (c) Detail of an emitter tip. (d) Detail of the black silicon surface treatment.

an emitter-to-extractor bias voltage and field enhancement at the emitter tip. For a large enough voltage, a stream of charged particles is emitted from the emitter tip. The emitters have a triangular-pyramid shape, except in the 502-emitter array where neighboring pyramids are partially merged for higher density [Fig. 3(b)]. The pyramids have submicrometer-sized tip radii [Fig. 3(c)] to provide large electric field enhancement and, thus, require a low startup voltage. The startup voltage in an externally fed electrospray emitter is given by [48]

$$
V_{\text {start }}=\sqrt{\frac{\gamma \cdot R}{\varepsilon_{o}}} \ln \left[\frac{2 G}{R}\right]
$$

where $\varepsilon_{o}$ is the electrical permittivity of free space, $\gamma$ is the surface tension of the liquid, $G$ is the emitter-to-extractor gap, $R$ is the emitter tip radius, and $2 G \gg R$.

The wicking material of the liquid supply system is black silicon [49]. The black silicon provides high hydraulic impedance to the electrospray emitter, allowing it to work in the pure ionic regime if fed with the ionic liquids $\mathrm{EMI}^{-\mathrm{BF}_{4} \text { or }}$ EMI-Im. Instead of a chlorine-based plasma as in [23], an $\mathrm{SF}_{6}$-based plasma recipe was used to create the black-silicon coating, following the procedure proposed by Jansen et al. [49]. The morphology of the black silicon that coats the emitters can be approximated by a set of micrometer-sized conic spikes that are separated by a distance $\varepsilon \sim 1-2 \mu \mathrm{m}$ [Fig. 3(d)]. The viscous flow across the black silicon can be modeled as the flow in an open capillary [50]. Therefore, the liquid flow within the black silicon can be modeled by the following 2-D version of Darcy's law:

$$
\vec{q}_{s}=-\frac{K_{\mathrm{ps}}}{\mu} \vec{\nabla} P
$$

where $P$ is the pressure, $K_{\mathrm{ps}}$ is the surface permeability (it has dimensions of a volume), $\mu$ is the viscosity of the fluid, and $\vec{q}_{s}$ is the volumetric surface flow rate flux. To estimate the hydraulic impedance of the black silicon, the emitter is modeled as a cone with semiangle $\theta=30^{\circ}$ (Fig. 4). The flow of liquid occurs between $z_{1}$, i.e., the base of the emission region 


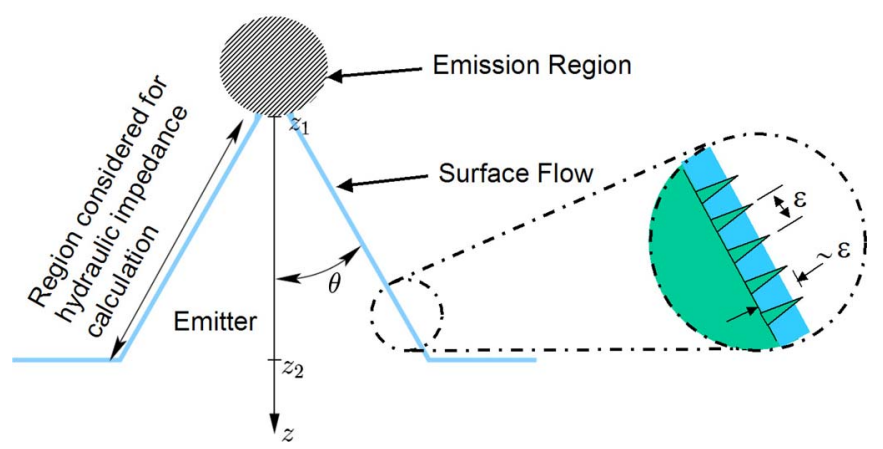

Fig. 4. Schematic of an emitter with black silicon, coated with ionic liquid. The liquid is transported within the surface of the emitter to the field-emission site at the emitter tip. The lateral surface of the emitter tip provides hydraulic impedance to the emission region.

$\sim 1 \mu \mathrm{m}$, and $z_{2}$, i.e., the height of the emitter $\sim 400 \mu \mathrm{m}$, within the black silicon. Applying (3) to the cone geometry results in the following relationship between the pressure drop and the surface permeability:

$$
\Delta P=-\frac{\mu}{K_{\mathrm{ps}}} \int_{Z_{1}}^{Z_{2}} \frac{Q}{2 \pi \cdot z \cdot \sin [\theta]} d z=-\frac{\mu \cdot Q \cdot \ln \left[z_{1} / z_{2}\right]}{2 \pi \cdot K_{\mathrm{ps}} \cdot \sin [\theta]}
$$

where $Q$ is the flow rate, which can be estimated from the emitter current $I$ as

$$
Q=\frac{I \cdot\langle M\rangle}{N_{\mathrm{AV}} \cdot e \cdot \rho}
$$

where $\langle M\rangle$ is the average molar mass of the emitted particles, $N_{\mathrm{AV}}$ is Avogadro's number, $e$ is the elementary charge, and $\rho$ is the density of the liquid. For the ionic liquid EMI-BF 4 , $I \sim 0.2 \mu \mathrm{A} \quad$ (see Section IV), $\langle M\rangle \sim 0.2 \mathrm{~kg} / \mathrm{mol}$, and $\rho=1333 \mathrm{~kg} / \mathrm{m}^{3}$ [51], resulting in $Q=3.1 \times 10^{-16} \mathrm{~m}^{3} / \mathrm{s}$. Using $\mu=0.0314 \mathrm{~Pa} \cdot \mathrm{s}$ for $\mathrm{EMI}^{-\mathrm{BF}_{4}}$ [51] and $\Delta P \sim 10 \mathrm{~Pa}$ [52], (4) results in $K_{\mathrm{ps}} \sim 1.86 \times 10^{-18} \mathrm{~m}^{3}$. Let us assume that the thickness of the liquid film is comparable to $\varepsilon$ (close up, Fig. 4). In viscous flow, for a given pressure gradient, the maximum velocity of the fluid is proportional to the square of the distance to the stationary wall. In the context of porous surface flow, this translates to $q_{s} / \varepsilon$ and, hence, $K_{\mathrm{ps}} / \varepsilon$ being proportional to the square of the porosity $\varepsilon^{2}$. Thus, we have

$$
K_{\mathrm{ps}} \propto \varepsilon^{3}
$$

Equation (6) predicts $\varepsilon \sim 1.3 \mu \mathrm{m}$, which is of the same order of the typical spike pitch value from SEM measurements. In our previous work [23], black silicon was found by trial and error as a suitable coating to make micromachined silicon emitters wettable by ionic liquids. The black-silicon morphology has a distribution that is tunable only to some degree by the plasma processing conditions, as shown by the AFM data [23]. Therefore, it is rather remarkable that the black silicon has the precise morphology to be used as hydraulic impedance in micromachined ionic liquid-fed electrospray ion sources. A substantially smaller hydraulic impedance in series with each emitter would result in emission of charged ionic liquid droplets

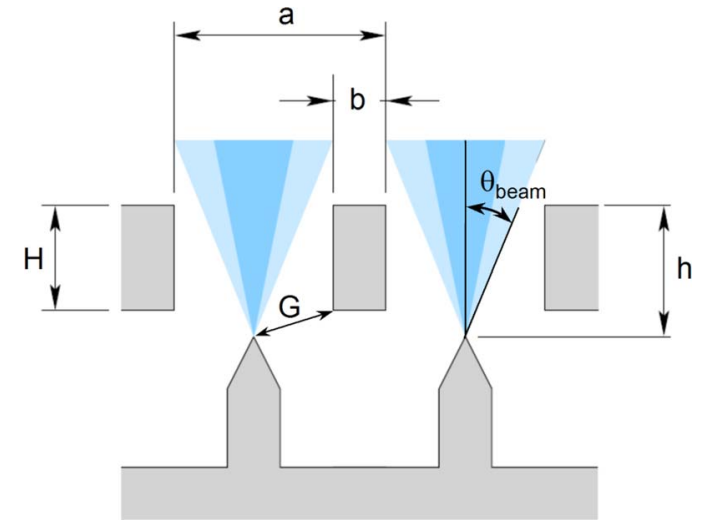

Fig. 5. Cross section of two adjacent electrospray emitters in operation. The size of the extractor slots and the emitter-to-extractor gap minimize beam interception by the extractor.

as other researchers have shown [33], with inherently smaller specific charge.

\section{B. Extractor}

The design of the extractor focuses on increasing the emitter density and lowering the extraction voltage. To increase the emitter density, the extractor slot width and slot-to-slot separation should be as small as possible. However, the extractor must have structural integrity against electrostatic loads and avoid beam interception. The dimensions that describe the extractor slot are the extractor thickness $H$, the slot pitch $a$, the width of material between two consecutive slots $b$, and the vertical separation $h$ between the emitter tips and the top surface of the extractor (Fig. 5). For a given $h$ and an electrospray beam divergence semiangle $\theta_{\text {beam }}$, the nonimpingement condition is

$$
\frac{a-b}{2 h}>\tan \left[\theta_{\text {beam }}\right] \text {. }
$$

In the implemented extractor $H=200 \mu \mathrm{m}, a=750 \mu \mathrm{m}$, $b=200 \mu \mathrm{m}$, and $h=325 \mu \mathrm{m}$, which yields no particle interception for $\theta_{\text {beam }} \leq 40^{\circ}$. The measured plume divergence of an electrospray emitter operating in single Taylor cone mode with ionic liquids [21], [23] is smaller than this value.

The emitter-to-extractor gap $G$ is given by

$$
G=\sqrt{(a-b)^{2} / 4+(h-H)^{2}} .
$$

Equation (8) predicts $G=302 \mu \mathrm{m}$ for the dimensions implemented in the MEMS electrospray array. With this emitter-toextractor gap, an electrospray emitter with a micrometer-sized tip radius that uses $\mathrm{EMI}^{-\mathrm{BF}_{4}}(\gamma=0.052 \mathrm{~N} / \mathrm{m}$ [53]) has a startup voltage of about $491 \mathrm{~V}(2)$, which is in agreement with the experimental data (see Section IV).

While gathering the data shown in Section IV, the MEMS electrospray array was biased at voltages up to $1.5 \mathrm{kV}$ in both polarities. The operational bias voltage level of the MEMS electrospray array is well below the electrical breakdown voltage of the emitter-to-extractor gap. The ionic liquids EMI-BF $\mathrm{EF}_{4}$ and EMI-Im have a very low (so far nonmeasurable) vapor pressure. Therefore, a high-vacuum condition at the gap between the 
tip and the extractor is expected. For a small enough product of the vacuum level and the separation gap, the electrical breakdown of vacuum is dominated by field emission instead of the Townsend breakdown theory, i.e., the Paschen curve [54]. In the field emission model, the ability of a sharp tip to emit electrons at a given bias voltage depends on the workfunction $\phi$ of the emitter material and the field-enhancing capability of the emitter, denoted by the field factor $\beta$ [55]. The field factor is a function of geometric parameters such as the tip radii, the emitter-to-electrode gap, and the emitter-to-emitter separation. The field factor of the emitter is substantially larger than the field factor of the extractor. Therefore, vacuum breakdown due to field emission is only relevant when the emitter die is biased negative to the extractor. The field factor of the emitters of the MEMS electrospray array is such that they require substantially more bias voltage to field emit electrons than to produce electrospray [55], [56].

During the operation of the MEMS electrospray array, the extractor experiences electrostatic forces that attract it to the emitter die. The following structural analysis of the extractor models silicon as an isotropic elastic material. The choice of the extractor thickness comes from considering two effects of the electrostatic load. First, the deflection due to the electrostatic pressure should be smaller than one third of the gap between the emitter and the extractor to avoid pull-in [57]. Second, the extractor should withstand the stresses induced by the electrostatic load. The electrostatic pressure $P_{\mathrm{el}}$ is modeled under the assumption that the electric field acting on the extractor is similar to the field in a parallel-plate capacitor with an interelectrode separation of $d$, i.e.,

$$
P_{\mathrm{el}}=\frac{\varepsilon_{o} V^{2}}{2 d^{2}} .
$$

The deflection $\delta$ of a doubly clamped beam of length $L$, height $H$, and Young's modulus $E$ under the influence of a uniform pressure $P_{L}$ is [58]

$$
\delta=\frac{P_{L} \cdot L^{4}}{32 \cdot E \cdot H^{3}}
$$

The extractor is made of structural beams with spacing $a$ and width $b$. Assuming conservatively that the electrostatic pressure is concentrated at the structural beams yields $P_{L}=(a / b) P_{\mathrm{el}}$. The separation between electrodes $d$ is about $h-H$. Therefore, the estimated deflection of the beam is given by

$$
\delta=\frac{\varepsilon_{o} \cdot V^{2} \cdot L^{4}}{64 \cdot[h-H]^{2} \cdot E \cdot H^{3}} .
$$

The slots of the extractor are about $16 \mathrm{~mm}$ long. Using a Young's modulus for silicon of $145 \mathrm{GPa}$ [57] and a maximum voltage of $5000 \mathrm{~V}$, (11) predicts that the extractor of the electrospray array deflects $12.5 \mu \mathrm{m}$, which is $10 \%$ of the undeflected emitter-to-extractor vertical separation. In terms of stress analysis, the bending stress is the dominating failure factor. The maximum bending stress is [58]

$$
\sigma=\frac{P_{L} \cdot L^{2}}{2 H^{2}}=\frac{a \cdot \varepsilon_{o} \cdot V^{2} \cdot L^{2}}{4 b \cdot[h-H]^{2} \cdot H^{2}} .
$$

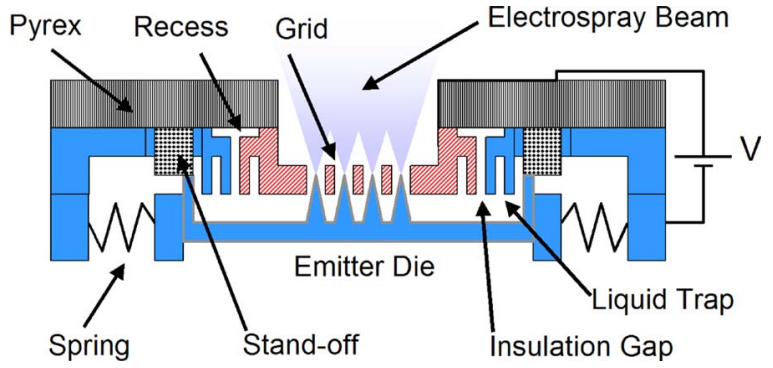

Fig. 6. Schematic of the electrical insulation system implemented in the MEMS electrospray array. The base of the electrical insulation system is a 0.5-mm-thick Pyrex layer, which also physically connects the two parts of the device with different polarities. The following three support features complete the electrical insulation system: 1) liquid traps surrounding the gap between the two parts of the device with opposite polarities; 2) a recess at the base of the gap between the two parts of the device with opposite polarities; and 3) a set of standoffs that allow varying the emitter-to-extractor gap.

For the extractor geometry and bias voltage equal to $5000 \mathrm{~V}$, the bending maximum stress is about $33 \mathrm{MPa}$, which is much less than the maximum bending stress for silicon, i.e., $1 \mathrm{GPa}[59]$.

\section{Electrical Insulation}

The MEMS electrospray array has a thorough set of features that provide electrical insulation between the extractor and the rest of the silicon-based portion of the electrospray array (Fig. 6). The main component of the electrical insulation system is a 0.5 -mm-thick Pyrex wafer, which can withstand a bias of up to $5 \mathrm{kV}$ between the extractor and the emitter die while having total leakage currents of less than $0.1 \mu \mathrm{A}$, which corresponds to the typical per-emitter startup current [46]. The Pyrex wafer is placed on top of the wafer stack rather than between the extractor and the springs to reduce the emitter-to-extractor gap and, thus, maximize the emitter density. This configuration allows many millimeters of Pyrex surface between the two equipotential zones of the electrospray array, reducing the risk of flashover without increasing the gap between the emitters and the extractor.

The length of Pyrex surface between the parts of the device with opposite polarities is increased by implementing recesses in the silicon substrate to which the Pyrex is bonded. The recesses also shadow some of the Pyrex-free surface, thus protecting it from contamination.

The electrical insulation system also implements a set of liquid traps. These traps are deep trenches on both sides of the insulation gap that separate the extractor slot region from the rest of the silicon-based portion of the electrode component. The wettability of the trenches was increased by the addition of mesoscaled sidewall features.

The electrical insulation system of the electrospray array is completed by a set of standoffs. Pyrex standoffs $500 \mu \mathrm{m}$ high were used in the work reported herein, although the specific material used has no engineering significance. The standoffs are inside $1.8 \mathrm{~mm} \times 2.6 \mathrm{~mm}$ holes etched into the electrode component [Fig. 1(b)], sticking out so that they contact both the bottom of the Pyrex wafer and the top of the emitter die ring [Figs. 1(c) and 2(a)]. The standoffs are integrated with 
the rest of the device at the end of the fabrication process using a reversible assembly procedure (see Section III-C). The standoffs are large enough to be handled with tweezers. The standoffs allow the vertical emitter-to-extractor gap to be adjusted without modifying either of the two parts of the MEMS electrospray array. The vertical positioning precision of the standoffs is controlled by the height precision of the standoffs and the roughness of the contact surfaces at the top and bottom of the standoffs. The implemented standoff system provides good vertical alignment because the standoffs are made from an anodic bondable Pyrex wafer scrap, the top surface of the emitter die ring is mirror-like fusion bondable silicon, and the bottom Pyrex surface is anodic bondable. Moreover, measurement of the height difference between the bottom of the emitter die and the bottom of the electrode component in four corners of the emitter die, using an interferometric microscope with $\pm 1 \mu \mathrm{m}$ resolution, yielded measurements identical to within $\pm 5 \mu \mathrm{m}$, which is of the order of the thickness total variation of a typical microfabrication wafer, and smaller than the variation in height between emitters.

\section{FABRICATION}

The MEMS electrospray array is fabricated using four 6-in wafers-one Pyrex wafer $500 \mu \mathrm{m}$ thick and three doubleside-polished (DSP) p-Si $\langle 100\rangle$ wafers $675 \pm 5 \mu$ m thick. The silicon wafers were purchased from Ultrasil, Hayward, CA, and the Pyrex wafer was obtained from Bullen Ultrasonics, Eaton, $\mathrm{OH}$. The emitter dies are fabricated using a silicon wafer, while the extractor components are fabricated using a threewafer stack. The Pyrex wafer is the top layer of the wafer stack and provides electrical insulation between the emitter die and the extractor component. The middle wafer of the stack has features such as the extractor slots, the liquid traps, the through-holes for the standoffs, and the electrical insulation gap. The bottom wafer of the stack contains the set of deflection springs that positions the emitter die. The electrospray array is fabricated using contact photolithography, low-temperature fusion bonding, anodic bonding, laser cutting, and deep reactive ion etching (DRIE). The complete process flow uses six optical masks: four masks are used to fabricate the common extractor component, and two masks are used to fabricate the emitter dies. Section III-A describes the process flow to fabricate the emitter dies. Section III-B describes the process flow to fabricate the extractor component. The process flow of the MEMS electrospray array is shown in Fig. 7.

\section{A. Fabrication of the Emitter Die}

The process flow starts with a DSP silicon wafer. First, both sides of the wafer are coated with a plasma-enhanced chemical vapor deposited (PECVD) silicon dioxide film. The front side of the wafer is coated with a 6- $\mu$ m-thick film, while the backside of the wafer is coated with a $4-\mu$ m-thick film. After the silicon dioxide deposition, the wafer is annealed at $950{ }^{\circ} \mathrm{C}$ in nitrogen. Next, the oxide films are patterned using a reactive ion etching (RIE) step with a $10-\mu$ m-thick spincoated AZ P4620 photoresist mask [Fig. 7(a)]. The oxide film

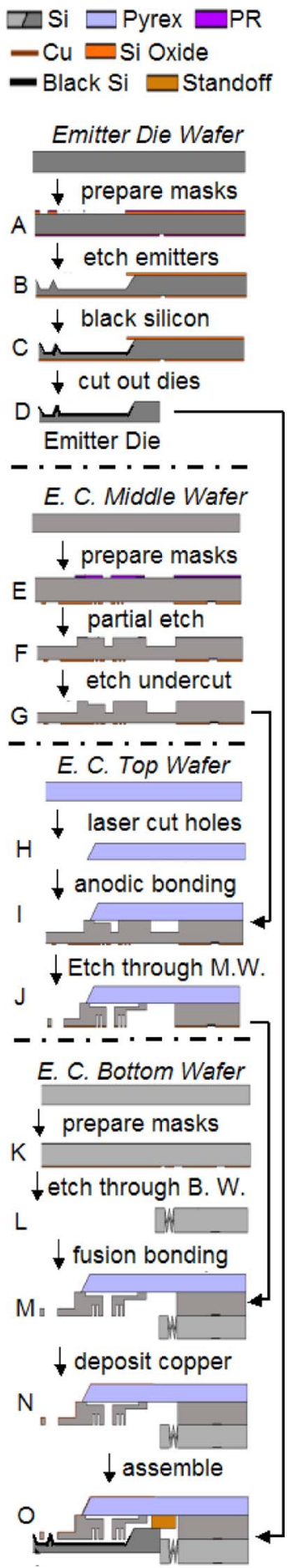

Fig. 7. MEMS electrospray array process flow.

on the topside of the wafer receives the features to fabricate the rows of emitters, while the oxide film on the backside of the wafer receives the features to extract the emitter dies from the substrate. The exposure of the topside photoresist film leaves alignment marks with enough contrast to align the backside exposure. After the silicon oxide films are etched, an alternation of DRIE and isotropic $\mathrm{SF}_{6}$ RIE steps similar to the work reported by Velásquez-García et al. [23], [60] defines the emitter geometry on the top of the wafer [Fig. 7(b)]. During the formation of the electrospray emitters, the wafer is mounted 
onto a handler wafer with photoresist to avoid breakage. After the emitters are etched, black silicon is formed on top of the emitters using an $\mathrm{SF}_{6}: \mathrm{O}_{2}$ plasma [61] [Fig. 7(c)]. Finally, the emitter dies are cut out from the wafer backside using DRIE. The emitter dies are kept in physical contact with the surrounding silicon using thin tethers that are broken with tweezers when the etch is completed. Without the tethers, dies would overheat once they were cut out from the wafer, allowing the plasma etch to undercut the oxide mask and destroy the die features. The dies are finally collected and dipped into a $49 \%$ hydrofluoric acid (HF) bath to strip the oxide masks [Fig. 7(d)].

\section{B. Fabrication of the Extractor Component}

1) Middle Wafer: The process flow starts with a DSP silicon wafer. First, both sides of the wafer receive a PECVD silicon dioxide film coating. The backside of the wafer receives a 6 - $\mu$ m-thick film, while the top of the wafer receives a $3-\mu \mathrm{m}$ thick film. The wafer is then annealed at $950{ }^{\circ} \mathrm{C}$ in nitrogen. After that, both sides of the wafer are spin coated with a $10-\mu \mathrm{m}-$ thick AZ P4620 photoresist film. The silicon oxide film on the top of the wafer is the first layer of a two-layer nested mask. The photoresist on the top of the wafer is exposed with the recess features that protect the Pyrex from surface contamination, the through-holes for the standoffs, and the electrical insulation gaps, as well as the features that reduce the thickness of the silicon substrate where the extractor slots are eventually etched. The exposure of the topside photoresist film leaves aligning marks with enough contrast to align subsequent photolithography and bonding steps. The photoresist film on the backside of the wafer is exposed with the features of the liquid traps, the extractor slots, and the electrical insulation gaps. The layout transferred to the backside also includes a set of recesses that let the deflection springs etched on the bottom wafer move freely once the bottom and middle wafers are bonded (see Section III-B3). After the photoresist is developed, the features in the photoresist are etched into the silicon dioxide films using an RIE step. The photoresist films are then stripped, and the top of the wafer is spin coated again with a $20-\mu \mathrm{m}$-thick AZ P4620 photoresist film. This photoresist film is the second layer of the two-layer nested mask. The photoresist film is exposed with the features of the through holes for the standoffs, the electrical insulation gaps, and the features that reduce the substrate thickness to fabricate the extractor slots. At this point, both the nested mask on top of the wafer and the hard mask on the bottom side are completed [Fig. 7(e)]. The top of the wafer is then etched by $450 \mu \mathrm{m}$ using a DRIE step to partially define the through-holes and thin out the extractor area [Fig. 7(f)]. After the DRIE step, the photoresist is stripped using oxygen plasma, and a short $20-\mu \mathrm{m}$ DRIE etch step is performed to carve the insulation recesses [Fig. 7(g)].

2) Top Wafer: The Pyrex wafer is cut using a Resonetics excimer laser at $248 \mathrm{~nm}$ [Fig. 7(h)]. Before cutting the substrate, both sides of the wafer are spin coated with thick photoresist to protect the wafer surface from materials redeposited during laser cutting. Through-holes at the location of the extractor slot region are etched. The wafer is then cleaned in a piranha bath to strip the protective photoresist coating.

3) Top/Middle Wafers: Once the Pyrex and the middle silicon wafer have been processed as previously described, the two wafers are prepared for bonding. The oxide on the top of the middle wafer is removed by quickly dipping the wafer into a $49 \% \mathrm{HF}$ bath. The silicon oxide etch rate of this bath is about $2.3 \mu \mathrm{m} / \mathrm{min}$. This step can be appropriately time controlled because of the large thickness difference between the top and backside oxide films on the middle wafer. Alternatively, an RIE step can be used instead of the HF dip to remove the top silicon oxide film. Once the top oxide film is stripped, a Piranha clean is performed on both wafers. The wafers are then anodic bonded [Fig. 7(i)]. Alignment of the two wafers is done by eye because only millimeter-scale precision is needed. The Pyrex/silicon wafer stack is then mounted with photoresist onto a handler wafer, with the Pyrex side down. The silicon side of the wafer stack is then etched with a DRIE step to create the insulation gap that effectively separates the extractor from the rest of the silicon wafer. This step also etches the recesses that allow the deflection springs to freely deflect, clears the through-holes for the standoffs, and defines the extractor slots [Fig. 7(j)]. Once the DRIE step is completed, the wafer stack is dismounted from the handler wafer with acetone, rinsed, and cleaned in an oxygen plasma.

4) Bottom Wafer: The process flow starts with a blank DSP silicon wafer. First, a $0.3-\mu \mathrm{m}$-thick thermal oxide film is grown on the wafer to protect its bonding surfaces. The top of the wafer is then coated with a 3- $\mu$ m-thick PECVD silicon oxide film. The substrate is then annealed at $950{ }^{\circ} \mathrm{C}$ in nitrogen. Next, the top of the wafer is spin coated with a $10-\mu \mathrm{m}$-thick AZ P4620 photoresist film, which is exposed with the features of the deflection springs. After the photoresist is developed, the features in the photoresist are etched into the silicon dioxide films using an RIE step [Fig. 7(k)]. Next, the springs are etched through the substrate using a DRIE step [Fig. 7(1)]. During the DRIE step, the wafer is mounted onto a handler wafer to prevent leakage of helium coolant. The set of springs is used in the final assembly of the electrospray array [46].

5) Top/Middle/Bottom Wafers: The full wafer stack is bonded using a low-temperature plasma-assisted fusion bonding step [62], [63] because the presence of the Pyrex wafer in the stack makes high-temperature annealing unfeasible. In preparation for bonding, the bottom wafer and the top/middle wafer stack are cleaned using a Piranha bath and then stripped of their oxide films using a $49 \%$ HF bath. The wafers are then placed in an RF oxygen plasma (30 s, 100 mtorr, $1 \mathrm{~kW})$. The substrates are then dipped in deionized water and spin dried. Finally, the substrates are aligned and pressed at room temperature while applying a force of $1.2 \mathrm{kN}$ [Fig. 7(m)]. The full wafer stack is then heated at $400{ }^{\circ} \mathrm{C}$ for $2 \mathrm{~h}$ in a nitrogen atmosphere with no pressing. The full wafer stack is die sawed to extract the individual common extractor components. Finally, a $1-\mu \mathrm{m}-$ thick copper film is sputtered onto the Pyrex side of the full wafer stack [Fig. 7(n)] using an aluminum shadow mask. The copper film is intended to make contact between the top surface of the full wafer stack and the extractor, allowing a bias voltage to be applied to the extractor. 


\section{Final Assembly of the MEMS Electrospray Array}

The standoffs are fabricated using a die saw and a wafer made of an arbitrary material that has the desired thickness. The standoffs are inserted into their corresponding holes in the electrode component. Then, the emitter die is twisted into place with a plastic tool that mates with assembly holes etched in the emitter die. This operation makes the springs of the extractor component align and clamp the emitter die [Fig. 7(o)]. Finally, pressure is applied to the die above each standoff to set the emitter-to-extractor gap. After assembly, the standoffs are held in slight compression between the emitter die and the Pyrex wafer. For disassembly, tweezers are used to pry up the emitter die and the standoffs. Features have been included on the extractor component to facilitate prying of the emitter die by allowing easy access to its edge.

\section{EXPERIMENTAL CHARACTERIZATION}

The MEMS electrospray array was tested with the ionic liquids EMI-BF ${ }_{4}$ and EMI-Im. The experimental characterization of the electrospray beam included current-voltage $(I-V)$ characteristics, time-of-flight (TOF) mass spectrometry, beam divergence, and imprints on a collector. The three array sizes described in Table I were tested. However, this section only reports the data from 216- and 502-emitter arrays as they consistently reached steady operation after an initial overwet phase. In contrast, the one-emitter arrays never reached steady operation, perhaps because of particle contamination on the emitter die. In more detail, SEMs of the emitter dies occasionally showed particle contamination, presumably from moving the devices between the cleanroom and the testing rig, and it is likely that some of these particles were emitting during the tests [61]. In addition, the variation in the emitter tip radius should significantly influence the emitter field enhancement, affecting particularly the emitter startup voltage (2). Particle contamination and emitter tip radius variation are also present on the larger arrays, but their impact is substantially diminished when estimating per-emitter performance.

\section{A. Electronic Instrumentation}

A Keithley 6514 and a Keithley 6517A electrometers were used for current measurements. An Agilent 54835A Infiniium oscilloscope was used to gather waveform data and to take voltage measurements. Pulses for the TOF experiments were provided by a BNC 555 pulse delay generator and were amplified with a DEIPVM-4210 output pulser module, which outputs pulses up to $\pm 950 \mathrm{~V}$. The emitter die voltage was supplied by a \pm 5 -kV Matsusada AMS-5B5, which was controlled by an Agilent 33220 A function generator. The bias voltage was accurate to within $20 \mathrm{~V}$. In the TOF experiments, a $3.5-\mathrm{kV}$ Acopian P03.5HN17 power supply was used to set the secondary electron suppression grid voltage, and a $+3.5-\mathrm{kV}$ Acopian P03.5HP17 power supply was used for the Einzel lens. A homemade high-speed current amplifier circuit based on a TI OPA655 operational amplifier was used to measure the highspeed TOF signal. The vacuum chamber was pumped down using two Varian TV-70 turbopumps. Finally, the chamber vac- uum was measured using a Varian IMG-100 inverted magnetron gauge.

\section{B. Testing Procedure}

About $0.1-1 \mathrm{~mm}^{3}$ of ionic liquid was delivered to the emitter die on the black-silicon-treated surface. The liquid spread across the black-silicon surface within a few minutes, coating the emitter array. Once wetted, the emitter die was assembled to the extractor component. After assembly, the MEMS electrospray array was mounted into a high-density polyethylene holder. A flexible clamp on the front side of the holder held the device in place and provided electrical contact to the extractor. Electrical contact to the emitters was made via the bottom wafer of the electrode component using a strip of copper tape attached to the polyethylene holder. Once the MEMS electrospray array was mounted, the holder was placed in a vacuum chamber that was pumped down to a base pressure below $1 \times 10^{-5}$ torr before starting the experiments. After the tests were conducted, the vacuum chamber was vented, and the emitter die was disassembled to be examined and cleaned afterward. It was confirmed experimentally that the emitter dies are reusable and that there was no apparent damage to the emitters after each run. Nevertheless, a small decrease in performance was observed upon refueling. The SEMs of tested emitter dies did not show any evidence of emitter sputter deterioration. However, the SEMs revealed that the some of the emitters were partially coated with a thin film similar to the collector imprints (see Section IV-H). This coating is probably due to the electrochemical effects present in the electrospray phenomenon [64]. Using energy-dispersive X-ray (EDX) spectroscopy, it was determined that the coating was formed by ions from the ionic liquid. SEMs of the tested emitter dies after the EDX spectroscopy showed the emitters unsputtered and with no coating, suggesting that such analysis readily removes the coating without damaging the emitters.

\section{Stability of Operation}

It was found experimentally that the electrospray array requires a higher startup voltage the first time it is turned on than during subsequent operation. The authors speculate that the extra voltage might be involved in completing the wetting of the emitters. Based on this assumption, every freshly started electrospray array was briefly biased at a voltage that resulted in a total collected current in the 10-mA range. After this conditioning process, the electrospray array would go through three distinctive phases of operation.

1) Overwet Phase: This phase occurs during the first few minutes of operation of the electrospray array, and it is characterized by unsteady emission, high extractor interception due to a larger beam divergence, and droplet emission (see Section IV-H).

2) Steady Phase: In this phase where the electrospray array exhibits a repeatable $I-V$ characteristic with very low extractor interception current. The experimental characterization of the steady phase is further discussed in this report.

3) Depletion Phase: This phase is characterized by low extractor interception current, but the current for a given voltage 


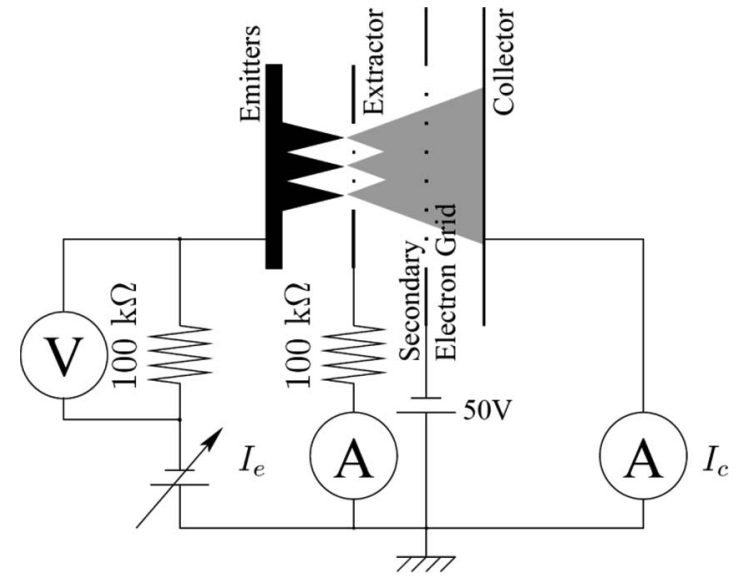

Fig. 8. $I-V$ characterization experimental setup.

slowly decreases compared to the emitted current in the steady phase. The authors speculate that the current is reduced due to depletion of the limited amount of liquid deposited at the beginning of the tests, i.e., there is no external liquid reservoir.

\section{I-V Characteristics: Steady Phase}

$I-V$ characteristics were obtained for the MEMS electrospray array while working in the steady phase. The current intercepted by the extractor was less than $1 \%$ of the emitted current over most of the operating range [46]. The experimental setup is shown in Fig. 8. In these experiments, the electrospray array was fired against a grounded collector plate. A secondary electron suppression grid was placed in front of the collector plate and biased to $+50 \mathrm{~V}$. The $I-V$ measurements were entirely computer controlled. Collector current, extractor current, emitter die voltage, and time were logged. Each data set was composed of three voltage sweeps to check for consistency of the measurements. Fig. 9(a) shows the per-emitter $I-V$ characteristics of three different array dies. There is a linear dependence between the bias voltage and the per-emitter current when the per-emitter current is above $100 \mathrm{nA}$, with a 1.2-nA/V slope. The slope is consistent across all the runs. However, the voltage at which the linear portion intersects the $x$-axis varies between 900 and $1400 \mathrm{~V}$ across the data sets. The highest per-emitter current observed was just over $1 \mu \mathrm{A}$, but at this high-current level, the device quickly runs out of liquid. The low-current behavior is shown in Fig. 9(b) using a semilog scale, which suggests that there is an exponential dependence on the emitted current with respect to the bias voltage as in [23], but the precise functional dependence is not clear. The overall shape of the characteristic is similar between different runs. The linear dependence of the emitted current on the bias voltage for high enough voltages suggests that at high current levels, the emission is controlled by a ballasting structure, presumably the black silicon. The electrospray emitters seem to behave analogously to a diode, where for a small voltage, there is an exponential dependence of the current on the bias voltage, but for large enough voltages, the resistance of the semiconductor determines the current, and hence, the current has a linear dependence with the voltage. However, the actual physical mechanism for current limitation and flow control that occurs in the electrospray emitters is not known. As a matter of fact, the physics of the pure ionic electrospray regime is a subject of active research [65]. The $I-V$ characteristics are symmetric if the magnitude of the per-emitter current versus bias voltage is plotted. If the startup voltage is defined as the voltage at which the per-emitter current is $5 \times 10^{-12} \mathrm{~A}$ (the peremitter noise floor), then startup voltages as low as $500 \mathrm{~V}$ were measured. Presumably, the electrospray array emits current at lower voltages. The startup value is slightly smaller than the minimum electrospray startup voltage previously reported in the literature $(550 \mathrm{~V})$ [27]. The startup voltage is probably representative of the sharper emitters of the array. In more detail, the emitter height variation and electrode sag due to electrostatic pulling should have little effect on the startup voltage uniformity across the array because the emitter-toextractor gap $G$ is almost two orders of magnitude larger than any of these two quantities. Furthermore, the startup voltage has a weak dependence on the electrode separation [see (2)]. However, the emitter tip radius has a distribution, which should significantly impact the startup voltage, as predicted by (2). Nonetheless, the emission across the array should be uniform at voltages that produce ballasted current emission.

\section{E. Startup Transient: Steady Phase}

In the experimental setup used to characterize the MEMS electrospray array, the startup transient in steady phase was observed by measuring the voltage across the collector resistor with the oscilloscope. In a typical startup transient, the collected current rises over a submillisecond timescale (Fig. 10). Then, the current exponentially decays toward a steady-state value, with a timescale on the order of $1 \mathrm{~s}$. At low currents, the overshoot is nearly nonexistent. At higher currents, the initial current peak can be over twice the steady-state current. As the current level increases, the duration of the transient slowly decreases. It is hypothesized that this behavior is due to the partial depletion of the liquid on the emitter surface after the emitter turns on. Initially, the liquid to be emitted is available directly in the emission region, but later on, a pressure gradient is necessary to draw liquid up the emitter surface. The partial depletion of liquid near the tip creates the pressure gradient, and the pressure gradient reduces the pressure in the liquid near the tip, reducing emission.

\section{F. Beam Angular Divergence: Steady Phase}

The angular spread of the electrospray beam was measured in the experiment depicted in Fig. 11 while operating in the steady phase. In this experiment, the collector plate was placed farther from the electrospray array than for the $I-V$ characteristics (about $7.5 \mathrm{~cm}$ ) to allow the device to be rotated around an axis $\left(y y^{\prime}\right)$ perpendicular to the direction of the slots. The beam travel distance is almost an order of magnitude larger than the length of the active area of the array perpendicular to the slots $(1 \mathrm{~cm})$, which allows modeling the MEMS array as a point source. Therefore, by rotating the device during operation, the emission current angular density $j_{b}(\theta)$ around $y y^{\prime}$ could be determined. 

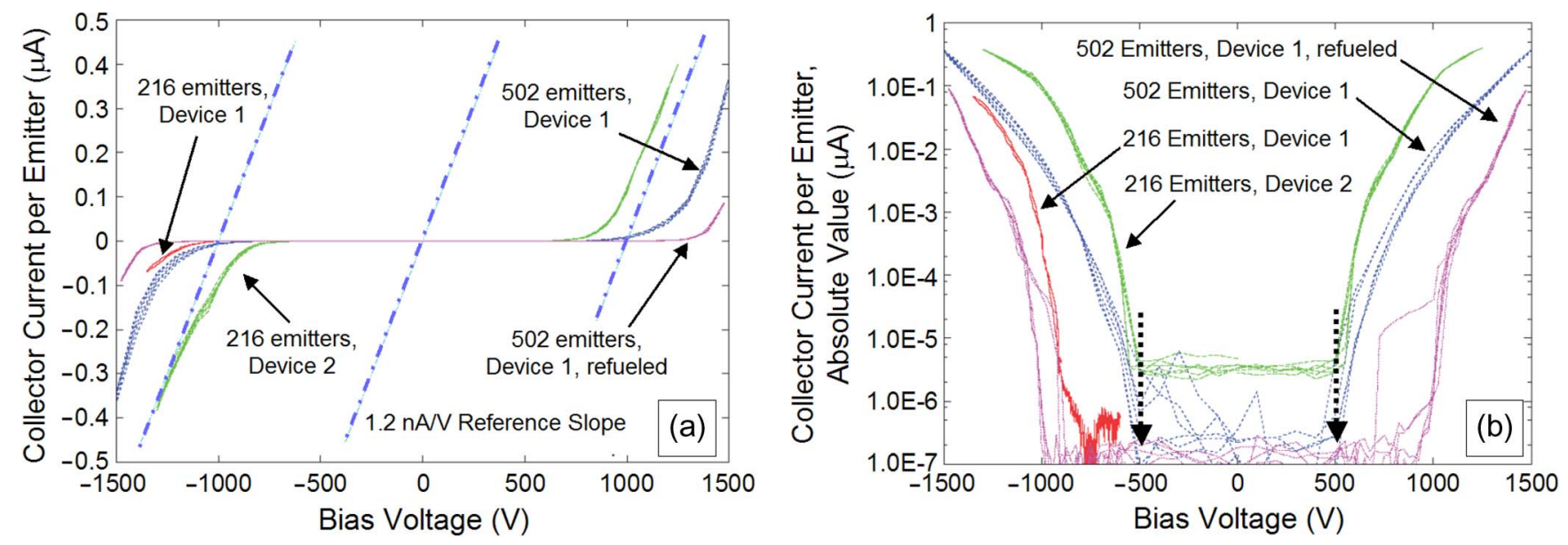

Fig. 9. $I-V$ characteristics using EMI-BF 4 . (a) High-current regime. (b) Low-current regime. The arrows on the low-current plot indicate the smallest startup voltage measured (i.e., $500 \mathrm{~V}$ ).

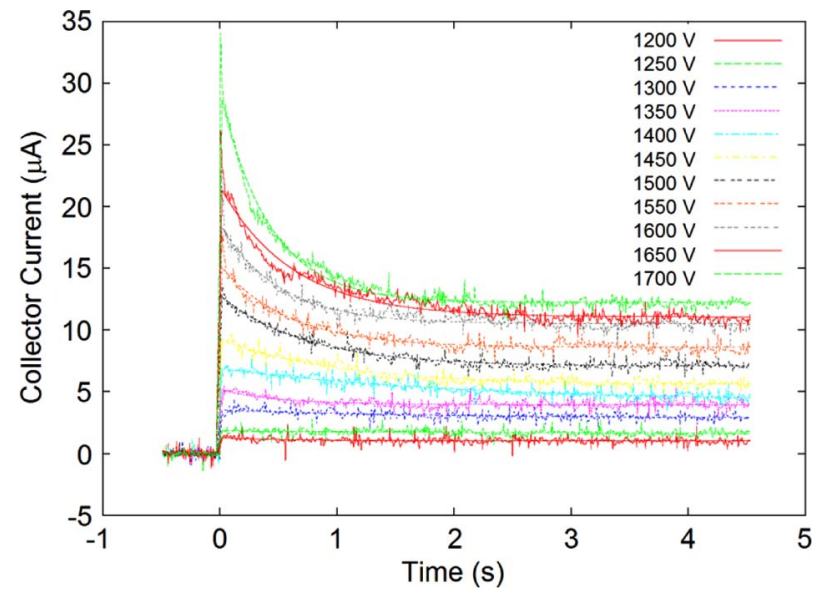

Fig. 10. Startup transients for different bias voltages.

Let $\theta_{r}$ be a measure of orientation around $y y^{\prime}$ with arbitrary origin. Then, $j_{b}\left(\theta_{r}\right) d \theta_{r}$ is the current emitted between the halfplanes, leaving $y y^{\prime}$ in the directions $\theta_{r}$ and $\theta_{r}+d \theta_{r}$. For an orientation $\theta_{r}$ of the electrospray array, the current collected by the collector plate is obtained by integrating the current densities between $\theta_{o}$ and $\theta_{o}+\Delta \theta_{r}$, where $\Delta \theta_{r}$ is the angular extent of the collector plate. Setting $\theta_{o}=0$ for simplicity, we obtain

$$
I_{c}\left(\theta_{r}\right)=\int_{0}^{\Delta \theta_{r}} j_{b}\left(\theta_{r}\right) d \theta_{r} .
$$

The data can be represented in a way that does not include the angular dimension of the plate by computing the current $I_{c}^{\infty}\left(\theta_{r}\right)$ that would be collected by a semiinfinite collector plate as follows ${ }^{1}$ :

$$
I_{c}^{\infty}\left(\theta_{r}\right)=\int_{0}^{\infty} j_{b}\left(\theta_{r}\right) d \theta_{r}=\sum_{k=0}^{\infty} I_{c}\left(\theta_{r}-k \cdot \Delta \theta_{r}\right) .
$$

\footnotetext{
${ }^{1}$ For simplicity, (14) is written as if angles extended to infinity without wrapping around. This approach is acceptable because $j_{b}(\theta)$ is only nonzero for a small range of angles, and therefore, the integration or the sums in (14) only need to extend until the current density goes to zero (i.e., until the measured current goes below the noise level).
}

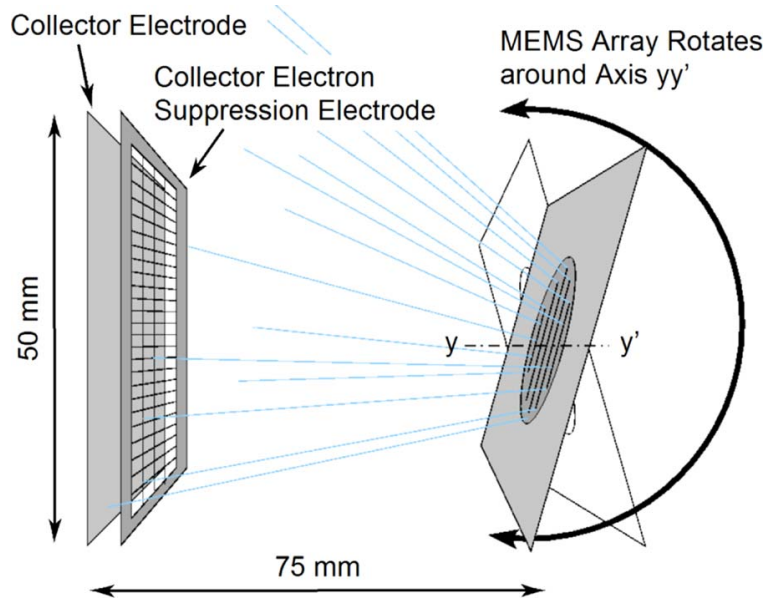

Fig. 11. Beam divergence characterization schematic.

Equation (14) allows $I_{c}^{\infty}\left(\theta_{r}\right)$ to be directly computed from the experimental data for $I_{c}\left(\theta_{r}\right)$. The corresponding plot is shown in Fig. 12(a). The plot reveals a variation of about $20^{\circ}$ on the direction of the maximum beam density as voltage is varied. The angular extent of the beam can be deduced from Fig. 12(b), in which the origin has been offset so that the zero-degree angle corresponds to $50 \%$ of the emitted current. For example, the angle at which the plot rises above 25\% indicates that 50\% of the current is contained between a semiangle of $5^{\circ}$ and $10^{\circ}$. Similarly, $90 \%$ of the current is contained within a semiangle of $10^{\circ}-20^{\circ}$. The angular distribution of the emissions in the other direction could not be characterized because of geometrical constraints.

\section{G. TOF Measurements in the Steady Phase}

Mass spectrometry of the electrospray beam was conducted using the TOF technique. The testing setup is shown in Fig. 13. The setup is similar to the one shown in Fig. 8, but now, the collector plate has been placed much farther from the MEMS electrospray array. The electrospray beam is passed through a 1/2-in aperture and then collimated by an Einzel electrostatic lens. The beam then passes through a gate that can be used to 

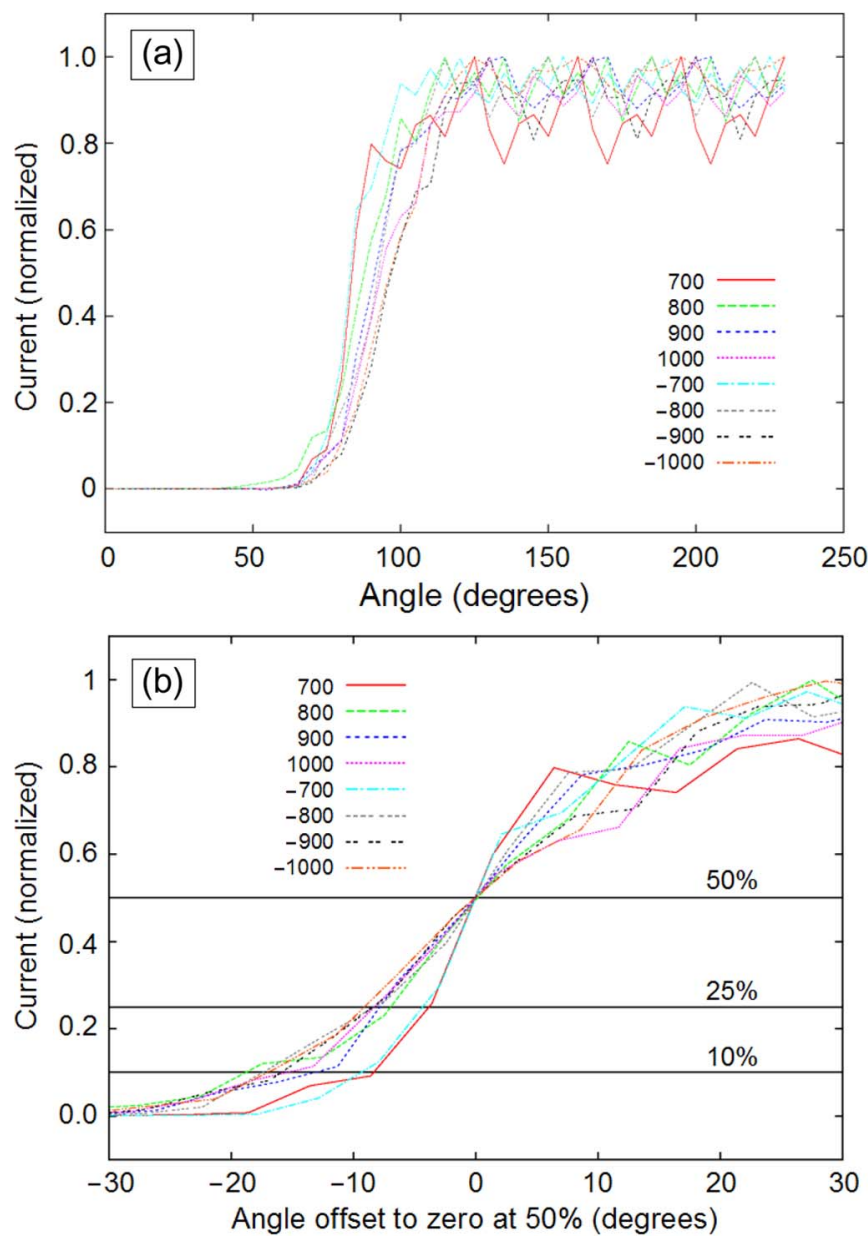

Fig. 12. Normalized current versus device orientation, extrapolated to a semiinfinite collector plate. With common origin for (a) all plots and (b) offset to $50 \%$ current at a zero-degree angle.

suddenly interrupt the beam by using a 950-V pulse. A collector plate intercepts the transmitted beam about $80 \mathrm{~cm}$ downstream from the gate. TOF measurements were taken by switching the gate and looking at the time delay in the current transient on the collector plate. Each data set shown in this section is the average of 1024 events. The data were processed to infer the mass $m$ of the corresponding charged particle, assuming that the particle charge is the elemental charge $e$ and that there is negligible voltage drop across the device when biasing a voltage $V$. With these assumptions, an energy balance allows the species mass to be calculated by

$$
m=\frac{2 \cdot e \cdot V \cdot t^{2}}{L_{i}^{2}}
$$

where $L_{i}$ is the gate-to-collector distance, and $t$ is the TOF.

Two ionic liquids were used with the MEMS electrospray

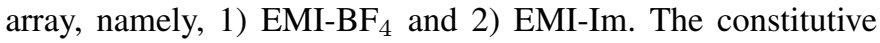
monomers are $\mathrm{EMI}^{+}=111.2 \mathrm{amu}, \mathrm{BF}_{4}^{-}=86.8 \mathrm{amu}$, and $\mathrm{Im}^{-}=280.2 \mathrm{amu}$. The pulsed TOF mass spectrometry of the beam coming out of the electrospray array using EMI-BF 4 is shown in Fig. 14. The data prove that for both polarities, the MEMS electrospray array operates in the pure ionic regime using $\mathrm{EMI}^{-\mathrm{BF}_{4}}$. The electrospray beam is mostly composed of the

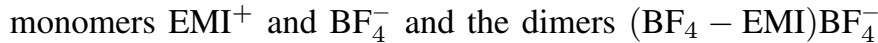
and $\left(\mathrm{BF}_{4}-\mathrm{EMI}\right) \mathrm{EMI}^{+}$. No droplets were detected in the TOF experiments with $\mathrm{EMI}_{-\mathrm{BF}_{4}}$. However, there is a small component of the beam made of trimers $\left(\mathrm{BF}_{4}-\mathrm{EMI}\right)_{2} \mathrm{BF}_{4}^{-}$and $\left(\mathrm{BF}_{4}-\mathrm{EMI}\right)_{2} \mathrm{EMI}^{+}$. In the negative polarity, the monomer current is about twice the dimer current. In the positive polarity, the monomer and dimer currents are about the same. The relative proportions of the species that compose the beam for each polarity were consistent across the various measurements to within $10 \%$.

The nonpulsed TOF mass spectrometry of the beam coming out of the electrospray array using EMI-Im is shown in Fig. 15. EMI-Im is an interesting substitute for the ionic liquid EMI-BF $F_{4}$ because it has lower surface tension, which should imply lower startup voltages [66], and also because it does not have a fluorine-based ion that might cause longterm etching of the silicon electrospray emitters. As in the previous TOF experiment, the data prove that using EMI-Im, the MEMS electrospray array operates in the pure ionic regime for both polarities. In this case, the electrospray beam is mainly composed of the monomers $\mathrm{EMI}^{+}$and $\mathrm{Im}^{-}$and the dimers $(\mathrm{Im}-\mathrm{EMI}) \mathrm{Im}^{-}$and $(\mathrm{Im}-\mathrm{EMI}) \mathrm{EMI}^{+}$. No droplets were detected in the TOF experiments using EMI-Im. However, there is a small component of the beam made of trimers $(\mathrm{Im}-\mathrm{EMI})_{2} \mathrm{Im}^{-}$ and $(\mathrm{Im}-\mathrm{EMI})_{2} \mathrm{EMI}^{+}$, as well as the tetramers $(\mathrm{Im}-\mathrm{EMI})_{3} \mathrm{Im}^{-}$ and $(\mathrm{Im}-\mathrm{EMI})_{3} \mathrm{EMI}^{+}$.

\section{H. Collector Imprints}

Silicon collectors were used as imprint targets. Two tests were conducted, namely, 1) a high-voltage test and 2) a lowvoltage test. In the high-voltage test, bias voltages up to $2500 \mathrm{~V}$ were applied, and total array currents up to $70 \mu \mathrm{A}$ were observed. Fig. 16(a) shows a typical target after firing the electrospray array in the high-voltage test. Thirteen rows are clearly visible, corresponding to the 13 rows of emitters of the emitter die. Microscopic examination of the imprints [Fig. 16(b)] reveals that each row is made up of patterns roughly spaced $500 \mu \mathrm{m}$ apart, which presumably correspond to each one of the emitters. These patterns have a roughly threefold symmetry, which is suggestive of the threefold symmetry of the ridgelines on the emitters.

The imprints in the low-voltage test are strikingly different [Fig. 16(c)]. In the low-voltage test, the bias voltage was increased until a total emitted current of a few hundred nanoamperes was measured. Rows of well-defined circles that are 250-350 $\mu \mathrm{m}$ in diameter were obtained (Table II). Many of the circles contain darker concentric dots up to $200 \mu \mathrm{m}$ in diameter, and each circle is surrounded by a roughly circular halo that is $500 \mu \mathrm{m}$ in diameter. The measured steady-state beam divergence semiangle that contains $90 \%$ of the current emitted by the electrospray array (i.e., $20^{\circ}$ ) is about as large as the corresponding semiangle for the typical maximum clean circle of the collector imprints (i.e., $21^{\circ}$ ). This result suggests that the beam divergence of the array is representative of the beam divergence of the individual emitters. As a matter of fact, the beam divergence of an ionic liquid electrospray emitter is basically constant along the beam axis [67] because the 


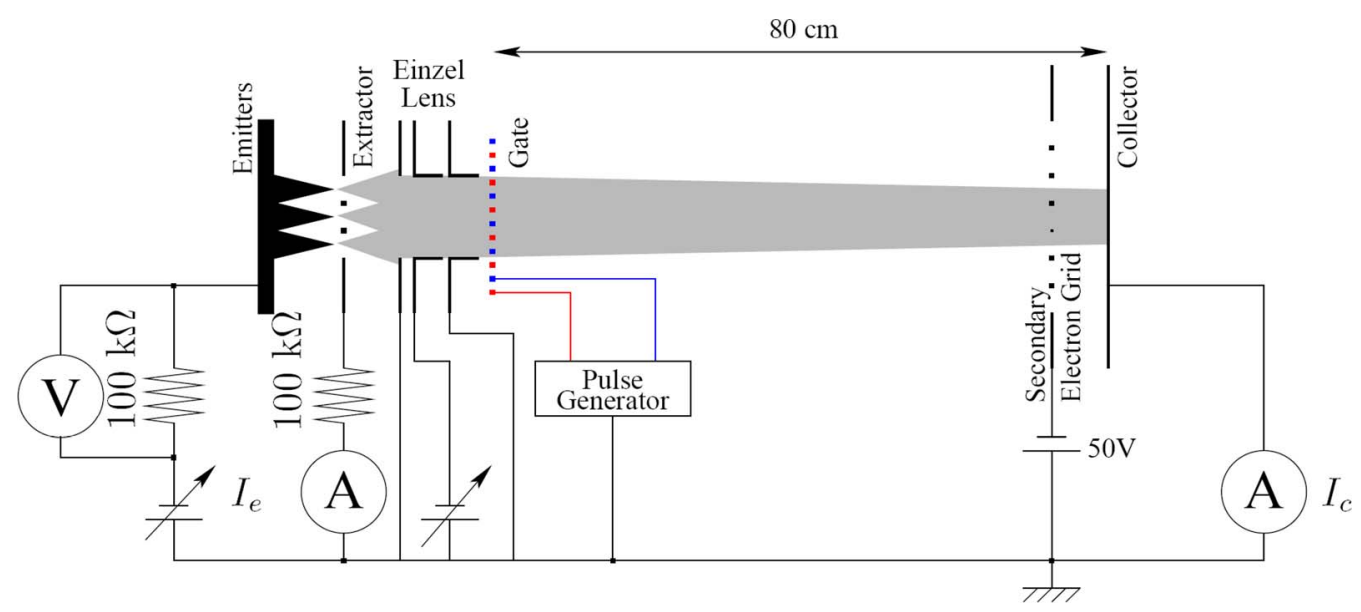

Fig. 13. TOF experimental setup.

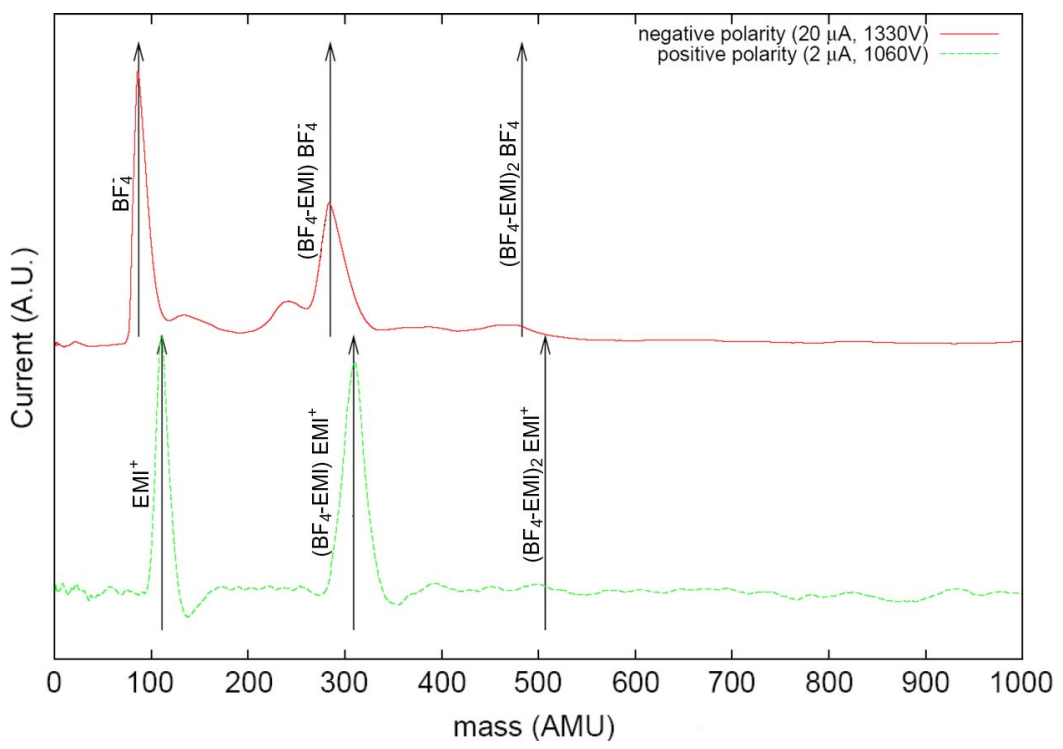

Fig. 14. TOF mass spectrum of the emitted beam using EMI-BF 4 .

Coulomb interactions within the spray are only important when the charge density is large, i.e., near the Taylor cone apex. As the spray travels, its charge density decreases due to the beam divergence. When the beams of adjacent emitters overlap, the charge density should still be low enough to produce no significant Coulomb interaction.

To analyze the low-voltage deposits, both SEMs and EDX spectroscopy of the target were conducted. The SEMs suggested that the central part of the imprints have some topography on the micrometer scale. The area outside the halo did not have this topography. EDX spectroscopy gathers information of the imprint within about $1 \mu \mathrm{m}$ of the surface. The EDX analysis reveals carbon and fluorine in the central spot, but no silicon, confirming that the spot has a micrometer-scale thickness. Around the central spot, there is an area with pure silicon (the halo), and further out, a weak fluorine and carbon signal reappears. The imprints could be explained by the following hypothetical sequence of events (Fig. 17). In the overwet phase, droplets are emitted and form a deposit on the target. The deposit is thicker toward its center. In the steady phase, pure ion emission occurs. The ions sputter away the deposited material over a circular region, getting through the thinner parts before the thicker parts. At higher current levels, the ion beam is broader, and the deposited material is completely removed. The ion energy appears not to be large enough to produce significant etching of the underlying silicon over the duration of these experiments.

\section{Applications of the Electrospray Array}

The experimental data suggests a number of tentative applications for the MEMS electrospray array ion source. All the proposed applications exploit the great versatility of electrospray. However, the applications are restricted to the use of the device with ionic liquids. First, the device could be used as a coating head. The imprints show that the beam divergence at high current is enough to produce overlap between the imprints of consecutive emitter rows. This way, time-controlled coating of a certain ionic liquid ion could be applied to a surface to functionalize it. Second, the device could be used as a print head, where a surface is selectively coated with a certain ionic liquid ion using a preestablished pattern. As a reference, both dropletemitting electrospray arrays [28] and ion-emitting electrospray arrays [23] have been shown to produce fine imprint patterns 


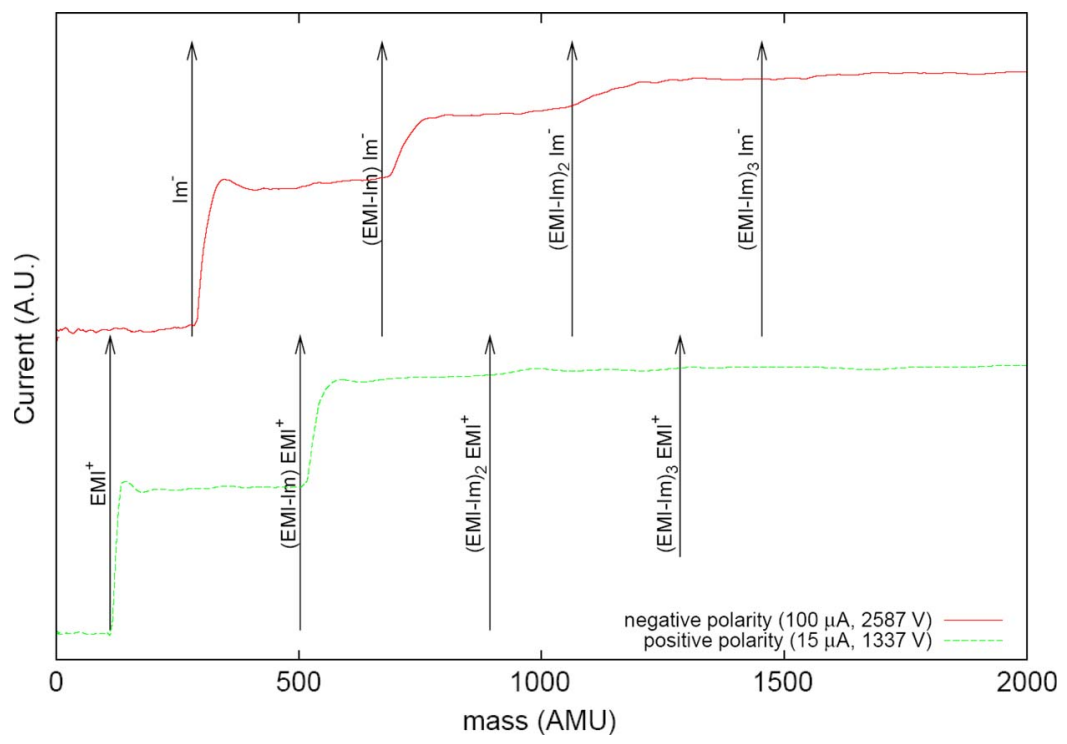

Fig. 15. TOF mass spectra of the emitted beam using EMI-Im.
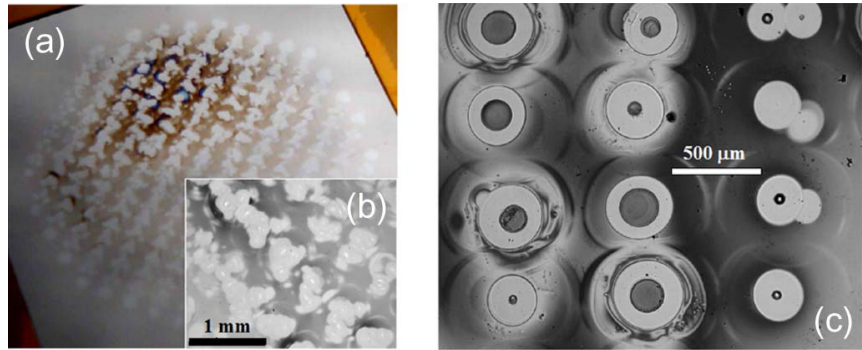

Fig. 16. (a) Collector imprints on a silicon target in the high-voltage test. (b) Detail of the high-voltage imprint patterns. (c) Collector imprints on a silicon target in the low-voltage test.

TABLE II

Metrology of THE LOW-Voltage IMPRINTS Using EMI-BF 4 AND PREVIOUSLY REPORTED WORK

\begin{tabular}{|c|c|c|}
\hline Imprint Feature & Radius $(\boldsymbol{\mu m})$ & Emission semi-angle $\left(^{\circ}\right)$ \\
\hline Inner circle (max) & 200 & 12 \\
\hline Clean circle (small) & 250 & 15 \\
\hline Clean circle (large) & 350 & 21 \\
\hline Outer halo & 500 & 29 \\
\hline Pencils in [23] & 35 & 9.5 \\
\hline Volcanoes in [23] & $-^{*}$ & 14.1 \\
\hline
\end{tabular}

${ }^{*}$ Volcano imprints resemble an 8-point sun corona with an internal non-imprinted halo. Therefore, these imprints do not have a characteristic radius that can be directly compared with the other imprint radii listed in Table II.

(i.e., lines of $70 \mu \mathrm{m}$ dots spaced $130 \mu \mathrm{m}$ and matrices of $70 \mu \mathrm{m}$ dots spaced $312 \mu \mathrm{m}$, respectively) without using collimating ion optics. For a printing application, the MEMS electrospray array should have an Einzel lens or ion optics to collimate the ion beam and, thus, achieve a smaller spot size than what was found in the experiment. The system would also include a precision stage positioning system. Third, the low-current imprint

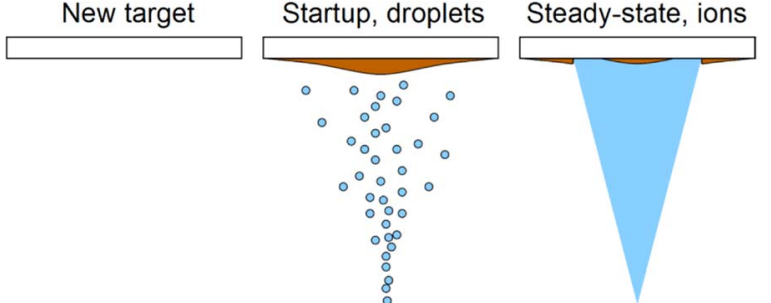

Fig. 17. Schematic of the proposed low-voltage imprint process.

experiments suggest that the ion beam is energetic enough to produce etching/sputtering in some materials. In this application, it would be important to understand and avoid the overwet phase, or to intercept its emissions with a shutter, so the ions can hit the surface instead of the droplet deposit. With a large enough bias voltage, the Taylor cone evolves into the highly stressed multicone regime, which has substantially less stability and more beam divergence [68]. Therefore, it seems unfeasible to etch silicon using ionic liquids with an extractor-only MEMS electrospray array ion source. However, we speculate that in the case of EMI-BF 4 , it could be possible that the fluorine-based ion could be accelerated using ion optics to gain enough energy to etch/sputter silicon. Finally, the properties of the electrospray beam (i.e., low polydispersity, small divergence, bipolar emission, low ionization losses, and output stability), united to the simplicity of the electrospray array architecture, makes this MEMS device attractive for nanosatellite propulsion applications [69]. The discussion of the application of the MEMS electrospray array for propulsion applications is beyond the scope of this paper and is found elsewhere [70].

\section{CONCLUSION}

The design, fabrication, and experimental characterization of a MEMS electrospray array that uses the ionic liquids EMI-BF and EMI-Im has been reported. The device is the first reported fully microfabricated planar array of externally fed electrospray emitters with integrated extractor that is shown to operate in 
the pure ion emission regime. Each MEMS electrospray array is composed of the following two parts: 1) an emitter die with as many as 502 emitters in $1.13 \mathrm{~cm}^{2}$ and 2) an extractor component that provides electrical insulation, alignment, and a common bias voltage to the emitter array. The emitters are coated with black silicon, which acts as a wicking material to transport the liquid to the emitter tips. The extractor and emitter die are assembled using a set of DRIE-patterned deflection springs. Experimental characterization of the electrospray beam includes $I-V$ characteristics, TOF mass spectrometry, beam divergence, and imprints on a collector. The reported data show that with ionic liquids EMI-BF 4 and EMI-Im, the electrospray array works in the pure ion emission regime for both polarities, emitting ions with a bias voltage as little as $500 \mathrm{~V}$. The data suggest that the MEMS electrospray array ion source could be used in applications such as coating, printing, etching, and nanosatellite propulsion.

\section{ACKNOWLEDGMENT}

The MEMS electrospray array was fabricated using the MIT Microsystems Technology Laboratories (MTL) facilities. The authors would like to thank the MTL staff for their invaluable support.

\section{REFERENCES}

[1] G. I. Taylor, "Disintegration of water drops in an electric field," Proc. $R$. Soc. Lond., vol. 280, pp. 383-397, Jul. 1964.

[2] J. Fernandez de la Mora, "The current emitted by highly conductive Taylor cones," J. Fluid Mech., vol. 260, pp. 155-184, 1994.

[3] M. Gamero-Castano and V. Hubry, "Electrospray as a source of nanoparticles for efficient colloid thrusters," J. Power Propuls., vol. 17, no. 5, pp. 977-987, Sep. 2001.

[4] I. Romero-Sanz, R. Bocanegra, J. Fernandez de la Mora, and M. Gamero-Castano, "Source of heavy molecular ions based on Taylor cones of ionic liquids operating in the pure ion evaporation regime," J. Appl. Phys., vol. 94, no. 5, pp. 3599-3605, Sep. 2003.

[5] P. Lozano and M. Martínez-Sánchez, "Experimental measurements of colloid thruster plumes in the ion-droplet mixed regime," presented at the 38th AIAA/ASME/SAE/ASEE Joint Propulsion Conf. Exhib., Indianapolis, IN, 2002, Paper AIAA 2002-3814.

[6] J. D. Regele, M. J. Papac, M. J. A. Rickard, and D. Dunn-Rankin, "Effects of capillary spacing on EHD spraying from an array of cone jets," J. Aerosol Sci., vol. 33, no. 11, pp. 1471-1479, Nov. 2002.

[7] J. Ishikawa and T. Takagi, "Impregnated electrode-type liquid metal ion source," Vacuum, vol. 36, no. 11/12, pp. 825-831, Nov./Dec. 1986.

[8] J. B. Fenn, M. Mann, C. K. Meng, S. F. Wong, and C. M. Whitehouse, "Electrospray ionization for mass spectrometry of large biomolecules," Science, vol. 246, no. 4926, pp. 64-71, Oct. 1989.

[9] S. Kaiser, D. C. Kyritsis, P. Dobrowloski, M. B. Long, and A. Gomez, "The electrospray and combustion at the mesoscale," J. Mass Spectrom. Soc. Jpn., vol. 51, no. 1, pp. 42-49, 2003.

[10] W. Deng, J. F. Klemic, X. Li, M. A. Reed, and A. Gomez, "Liquid fuel microcombustor using microfabricated multiplexed electrospray sources," Proc. Combust. Inst., vol. 31, pp. 2239-2246, Jan. 2007.

[11] I. W. Lenggoro, K. Okuyama, J. Fernandez de la Mora, and N. Tohge, "Preparation of $\mathrm{ZnS}$ nanoparticles by electrospray pyrolysis," J. Aerosol Sci., vol. 31, no. 1, pp. 121-136, Jan. 2000.

[12] W. Deng, J. F. Klemic, X. Li, M. A. Reed, and A. Gomez, "Increase of electrospray throughput using multiplexed microfabricated sources for the scalable generation of monodisperse droplets," J. Aerosol Sci., vol. 37, no. 6, pp. 696-714, Jun. 2006.

[13] D. Li and Y. Xia, "Electrospinning of nanofibers: Reinventing the wheel?" Adv. Mater, vol. 16, no. 14, pp. 1151-1170, Jul. 2004.

[14] S. E. Law, "Agricultural electrostatic spray application: A review of significant research and development during the 20th century," J. Electrostat., vol. 51, pp. 25-42, 2001.
[15] E. Pugh, "Method and apparatus for coating articles," U.S. Patent $1855869,1932$.

[16] B. Hoyer, G. Sorensen, N. Jensen, D. B. Nielsen, and B. Larsen, "Electrostatic spraying: A novel technique for preparation of polymer coatings on electrodes," Anal. Chem., vol. 68, no. 21, pp. 3840-3844, 1996.

[17] L. F. Velásquez-García,General Doctoral Examination, Dept. Aeronautics Astronautics, MIT, Cambridge, MA, Dec. 2001, unpublished.

[18] S. N. Jayasinghe, M. J. Edirisinghe, and D. Z. Wang, "Controlled deposition of nanoparticles clusters by electrohydrodynamic atomization," Nanotechnology, vol. 15, no. 11, pp. 1519-1523, Nov. 2004

[19] G. L. R. Mair, "Theoretical determination of current-voltage curves for liquid metal ion sources," J. Phys. D, Appl. Phys., vol. 17, no. 11, pp. 2323-2330, Nov. 1984.

[20] S. P. Thompson and P. D. Prewett, "The dynamics of liquid metal ion sources," J. Phys. D, Appl. Phys., vol. 17, no. 11, pp. 2305-2321, Nov. 1984

[21] P. Lozano and M. Martinez-Sanchez, "Ionic liquid ion sources: Characterization of externally wetted emitters," J. Colloid Interface Sci., vol. 282, no. 2, pp. 415-421, Feb. 2005.

[22] A. Genovese, M. Tajmar, N. Buldrini, M. Scheerer, E. Semerad, and W. Steiger, "Indium FEEP multiemitter development and test results," presented at the 40th AIAA/ASME/SAE/ASEE Joint Propulsion Conf. Exhib., Fort Lauderdale, FL, 2004, Paper AIAA 2004-3620.

[23] L. F. Velásquez-García, A. I. Akinwande, and M. Martinez-Sanchez, "A planar array of microfabricated electrospray emitters for thruster applications," J. Microelectromech. Syst., vol. 15, no. 5, pp. 1272-1280, Oct. 2006.

[24] R. D. Rogers, "Materials science: Reflections on ionic liquids," Nature, vol. 447, no. 7147, pp. 917-918, Jun. 2007.

[25] W. C. Burson, Jr. and P. C. Herren, Jr., "Alternating current operation of a colloid source," J. Spacecr., vol. 8, no. 6, pp. 606-611, 1971.

[26] L. F. Velásquez-García, "The design, fabrication and testing of micro fabricated linear and planar colloid thruster arrays," Ph.D. dissertation, Dept. Aeronautics Astronautics, MIT, Cambridge, MA, May 2004.

[27] R. Krpoun and H. R. Shea, "A method to determine the onset voltage of single and arrays of electrospray emitters," J. Appl. Phys., vol. 104, no. 6, p. 064 511, Sep. 2008.

[28] L. F. Velásquez-García, A. I. Akinwande, and M. Martínez-Sánchez, "A microfabricated linear array of electrospray emitters for thruster applications," J. Microelectromech. Syst., vol. 15, no. 5, pp. 1260-1271, Oct. 2006.

[29] Y. Wang, J. Cooper, C. S. Lee, and D. L. DeVoe, "Efficient electrospray ionization from polymer microchannels using integrated hydrophobic membranes," Lab Chip, vol. 4, no. 4, pp. 263-267, Aug. 2004.

[30] M. D. Paine, S. Gabriel, C. G. J. Schabmueller, and A. G. R. Evans, "Realization of very high voltage electrode-nozzle systems for MEMS," Sens. Actuators A, Phys., vol. 114, no. 1, pp. 112-117, Aug. 2004.

[31] M. Tajmar, "MEMS indium FEEP thruster: Manufacturing study and first prototype results," presented at the 40th AIAA/ASME/ SAE/ASEE Joint Propulsion Conf. Exhib., Fort Lauderdale, FL, 2004, Paper AIAA-2004-3619.

[32] E. H. Cardiff, B. G. Jamieson, P. C. Norgaard, and A. B. Chepko, "The NASA GSFC MEMS colloidal thruster," presented at the 40th AIAA/ASME/SAE/ASEE Joint Propulsion Conf. Exhib., Fort Lauderdale, FL, Jul. 2004, AIAA 2004-3592.

[33] R. Krpoun, M. Raber, H. R. Shea, K. L. Smith, and J. P. Stark, "Design and fabrication of an integrated MEMS-based colloid micropropulsion system," presented at the Int. Electric Propulsion Conf., Florence, Italy, 2007, IEPC 2007-099.

[34] M. S. Alexander, J. Stark, and K. L. Smith, "Electrospray performance of microfabricated colloid thruster arrays," J. Power Propuls., vol. 22, no. 3, pp. 620-627, 2006

[35] J. Xiong, Z. Zhou, D. Sun, and X. Ye, "Development of a MEMS based colloid thruster with sandwich structure," Sens. Actuators A, Phys., vol. 117, no. 1, pp. 168-172, Jan. 2005.

[36] J. Xiong, D. Sun, Z. Zhou, and W. Zhang, "Investigation of the onset voltage for the design of a microfabricated colloid thruster," IEEE/ASME Trans. Mechatronics, vol. 11, no. 1, pp. 66-74, Feb. 2006.

[37] I. M. Lazar, J. Grym, and F. Foret, "Microfabricated devices: A new sample introduction approach to mass spectrometry," Mass Spectrom. Rev., vol. 25, no. 4, pp. 573-594, Jul./Aug. 2006.

[38] L. Licklider, X.-Q. Wang, A. Desai, Y.-C. Tai, and T. D. Lee, "A micromachined chip-based electrospray source for mass spectrometry," Anal. Chem., vol. 72, no. 2, pp. 367-375, 2000.

[39] S. Arscott, S. LeGac, C. Druon, P. Tabourier, and C. Rolando, "A planar on-chip micro-nib interface for nano ESI-MS microfluidic applications," J. Micromech. Microeng., vol. 14, no. 2, pp. 310-316, 2004. 
[40] J.-S. Kim and D. R. Knapp, "Miniaturized multichannel electrospray ionization emitters on poly(dimethylsiloxane) microfluidic devices," Electrophoresis, vol. 22, no. 18, pp. 3993-3999, Oct. 2001.

[41] W.-C. Sung, H. Makamba, and S.-H. Chen, "Chip-based microfluidic devices coupled with electrospray ionization mass spectrometry," Electrophoresis, vol. 26, no. 9, pp. 1783-1791, May 2005.

[42] P. Griss, J. Melin, J. Sjodahl, J. Roeraade, and G. Stemme, "Development of micromachined hollow tips for protein analysis based on nanoelectrospray ionization mass spectrometry," J. Micromech. Microeng., vol. 12, no. 5, pp. 682-687, Sep. 2002.

[43] J. Vila, P. Ginés, J. M. Pico, C. Franjo, E. Jiménez, L. M. Varela, and O. Cabeza, "Temperature dependence of the electrical conductivity in EMIM-based ionic liquids. Evidence of Vogel Tamman Fulcher behavior," Fluid Phase Equilib., vol. 242, no. 2, pp. 141-146, Apr. 2006.

[44] J. A. Widegren, E. M. Saurer, K. N. Marsh, and J. W. Magee, "Electrolytic conductivity of four imidazolium-based room-temperature ionic liquids and the effect of a water impurity," J. Chem. Thermodyn., vol. 37, no. 6, pp. 569-575, Jun. 2005.

[45] L. F. Velásquez-García, A. I. Akinwande, and M. Martínez-Sánchez, "Precision hand assembly of MEMS subsystems using DRIE-patterned deflection spring structures: An example of an out-of-plane substrate assembly," J. Microelectromech. Syst., vol. 16, no. 3, pp. 598-612, Jun. 2007

[46] B. Gassend, L. F. Velásquez-García, and A. I. Akinwande, "Precision in-plane hand assembly of bulk-microfabricated components for highvoltage MEMS arrays applications," J. Microelectromech. Syst., 2009, to be published. DOI: 10.1109/JMEMS.2008.2011115.

[47] C. M. Waits, N. Jankowski, and B. Geil, "Scalable electrospray components for portable power applications using MEMS fabrication techniques," in Proc. 25th Army Sci. Conf., 2006, 8 pp.

[48] P. D. Prewett and G. L. R. Mair, Focused Ion Beams From Liquid Metal Ion Sources. Hertfordshire, U.K.: Research Studies Press, 1991

[49] H. Jansen, M. deBoer, R. Legtenberg, and M. Elwenspoek, "The black silicon method: A universal method for determining the parameter setting of a fluorine-based reactive ion etcher in deep silicon trench etching with profile control," J. Micromech. Microeng., vol. 5, no. 2, pp. 115-120, Jun. 1995.

[50] L. A. Romero and F. G. Yost, "Flow in an open channel capillary," J. Fluid Mech., vol. 322, pp. 109-129, 1996.

[51] S. Zhang, X. Li, H. Chen, J. Wang, J. Zhang, and M. Zhang, "Determination of physical properties for the binary system of 1-ethyl3-methylimidazolium tetrafluoroborate $+\mathrm{H}_{2} \mathrm{O}$," J. Chem. Eng. Data, vol. 49, no. 4, pp. 760-764, 2004.

[52] J. A. Carretero-Benignos, "Numerical simulation of a single emitter colloid thruster in pure droplet cone jet mode," Ph.D. dissertation, MIT, Cambridge, MA, 2005.

[53] W. Martino, J. Fernandez de la Mora, Y. Yoshida, G. Saito, and J. Wilkes, "Surface tension measurements of highly conducting ionic liquids," Green Chem., vol. 8, no. 4, pp. 390-397, 2006.

[54] L. Barrs-Hibbe, C. Schrader, P. Sichler, T. Cordes, K.-H. Gericke, S. Büttgenbach, and S. Draeger, "Micro-structured electrode arrays: Highfrequency discharges at atmospheric pressure-Characterization and new applications," Vacuum, vol. 73, no. 3/4, pp. 327-332, Apr. 2004.

[55] R. Gomer, Field Emission and Field Ionization. New York: AIP, 1993.

[56] D. G. Pflug, "Modeling the effects of device scaling on field emitter array performance," M.S. thesis, MIT, Cambridge, MA, 1996.

[57] S. Senturia, Microsystem Design. New York: Springer-Verlag, 2000.

[58] W. C. Young, R. G. Budynas, and R. J. Roark, Roark's Formulas for Stress and Strain. New York: McGraw-Hill Professional, 2001.

[59] K.-S. Chen, A. Ayon, and S. M. Spearing, "Controlling and testing fracture strength of silicon on the mesoscale," J. Amer. Ceram. Soc., vol. 83, no. 6, pp. 1476-1484, 2000.

[60] B. Gassend, L. F. Velásquez-García, A. I. Akinwande, and M. MartinezSanchez, "Design and fabrication of complex needle-like structures using DRIE," submitted for publication.

[61] B. Gassend, "A fully microfabricated two-dimensional electrospray array with applications to space propulsion," Ph.D. dissertation, Dept. Elect. Eng. Comput. Sci., MIT, Cambridge, MA, Jun. 2007.

[62] P. Amirfeiz, S. Bengtsson, M. Bergh, E. Zanghellini, and L. Börjessond, "Formation of silicon structures by plasma-activated wafer bonding," J. Electrochem. Soc., vol. 147, no. 7, pp. 2693-2698, 2000.

[63] X. Zhang and J.-P. Raskinz, "Low-temperature wafer bonding optimal $\mathrm{O}_{2}$ plasma surface pretreatment time," Electrochem. Solid-State Lett., vol. 7, no. 8, pp. G172-G174, 2004.

[64] J. Fernandez de la Mora, G. J. Van Berkel, C. G. Euke, R. B. Cole, M. Martinez-Sanchez, and J. B. Fenn, "Electrochemical processes in electrospray ionization mass spectrometry," J. Mass Spectrom., vol. 35, no. 8, pp. 939-952, Aug. 2000.

[65] J. Fernandez de la Mora, "The fluid dynamics of Taylor cones," Annu. Rev. Fluid Mech., vol. 39, no. 1, pp. 217-243, 2007.

[66] T. Cruz Garza, "Optimizing wettability of externally wetted microfabricated silicon electrospray thrusters," M.S. thesis, Dept. Aeronautics Astronautics, MIT, Cambridge, MA, 2004.

[67] I. Romero-Sanz and J. Fernandez de la Mora, "Energy distribution and spatial structure of electrosprays of ionic liquids in vacuo," J. Appl. Phys., vol. 95, no. 4, pp. 2123-2129, Feb. 2004

[68] J. Perel, "Mechanisms of emitter surface damage during electrohydrodynamic colloid particle generation and acceleration," Phrasor Technol., Pasadena, CA, AFOSR TR 78-1026, 1978. AFOSR Contract F44620-75C-0056.

[69] J. Mueller, "Thruster options for microspacecraft: A review and evaluation of existing hardware and emerging technologies," presented at the 33rd AIAA/ASME/SAE/ASEE Joint Propulsion Conf. Exhib., Seattle, WA, 1997, AIAA-97-3058.

[70] B. Gassend, L. F. Velásquez-García, A. I. Akinwande, and M. MartínezSánchez, "A fully integrated microfabricated externally wetted electrospray thruster," presented at the 43rd AIAA/ASME/SAE/ASEE Joint Propulsion Conf., Cincinnati, OH, Jul. 8-11, 2007, AIAA-2007-5182.

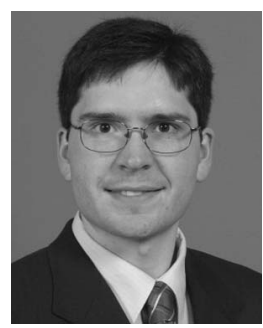

Blaise Gassend was born in Nice, France, in 1978. $\mathrm{He}$ received the Dipl.Ing. degree from the Ecole Polytechnique, Palaiseau, France, in 2001, and the M.S. degree in physical random functions and the $\mathrm{Ph} . \mathrm{D}$. degree in microfabricated electrospray thruster arrays from the Massachusetts Institute of Technology, Cambridge, in 2003 and 2007, respectively.

$\mathrm{He}$ is currently an Associate in Electrical and Semiconductor Practice with Exponent, Inc., Menlo Park, CA, where he consults on failure analysis and litigation support.

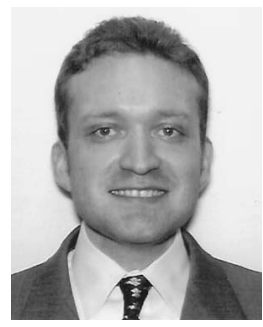

Luis Fernando Velásquez-García received the Mechanical Engineer (magna cum laude) and Civil Engineer (magna cum laude) degrees from the Universidad de Los Andes, Bogotá, Colombia, in 1998 and 1999, respectively, and the M.S. degree in aeronautics and astronautics and the $\mathrm{Ph} . \mathrm{D}$. degree in space propulsion from the Massachusetts Institute of Technology (MIT), Cambridge, in 2001 and 2004, respectively

In 2004, after completing his studies, he became a Postdoctoral Associate with the Microsystems Technology Laboratories, MIT, where he has been a Research Scientist since 2005. $\mathrm{He}$ is an expert in microfabrication and nanofabrication technologies. His research focuses on the application of microfabrication and nanofabrication technologies to propulsion, analytical, and power conversion systems. $\mathrm{He}$ has conducted research on microtechnologies and nanotechnologies applied to electrospray arrays, carbon-nanotube-based devices, 3-D packaging, mass spectrometry, propulsion, and chemical reactors. He is the author of more than ten journal publications and 25 conference proceedings works. He is the holder of six patents on MEMS technologies.

Dr. Velásquez-García is a member of Sigma Xi. 


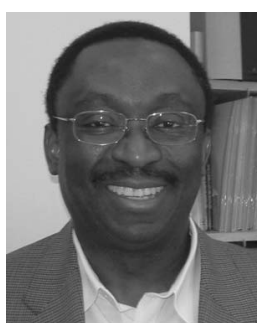

Akintunde Ibitayo (Tayo) Akinwande received the B.Sc. degree in electrical and electronic engineering from the University of Ife, Ife, Nigeria, in 1978, and the M.S. and Ph.D. degrees in electrical engineering from Stanford University, Stanford, CA, in 1981 and 1986, respectively.

In 1986, he joined Honeywell International, Inc., Morristown, NJ, where he initially conducted research on GaAs complementary FET technology for very-high-speed and low-power signal processing. He later joined the Si Microstructures Group, where he conducted research on pressure sensors, accelerometers, and thin-film field emission and display devices. In January 1995, he joined the Microsystems Technology Laboratories, Massachusetts Institute of Technology (MIT), Cambridge, where his research focuses on microfabrication and electronic devices, with particular emphasis on smart sensors and actuators, intelligent displays, large-area electronics (macroelectronics), field emission and field ionization devices, mass spectrometry, and electric propulsion. He was a Visiting Professor with the Department of Engineering and an Overseas Fellow with Churchill College, Cambridge University, Cambridge, U.K., in 2002 and 2003. He is currently a Professor with the Department of Electrical Engineering and Computer Science, MIT. He is the author of more than 100 journal publications and is the holder of numerous patents in MEMS, electronics on flexible substrates, and display technologies.

Prof. Akinwande was the recipient of a 1996 National Science Foundation CAREER Award. He is currently a member of the IEEE Nanotechnology Council. He has served on a number of Technical Program Committees for various conferences, including the Device Research Conference, the IEEE International Electron Devices Meeting, the IEEE International Solid-State Circuits Conference, the International Display Research Conference, and the International Vacuum Microelectronics Conference.

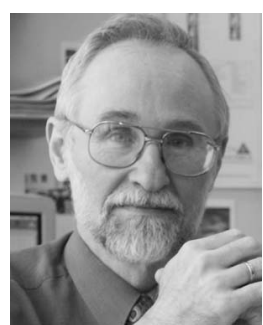

Manuel Martínez-Sánchez received the Aeronautical Engineer's degree from the Polytechnic University of Madrid, Madrid, Spain, in 1967, and the $\mathrm{Ph} . \mathrm{D}$. degree from the Massachusetts Institute of Technology (MIT), Cambridge, in 1973.

He is currently a Professor with the Department of Aeronautics and Astronautics, MIT. His research and teaching are focused on space propulsion, after having looked into MHD power generation and other energy-related topics in the past. $\mathrm{He}$ is the author of about 30 journal papers and 120 conference proceedings works.

Dr. Martínez-Sánchez is a Corresponding Member of the Spanish Academy of Engineering. 